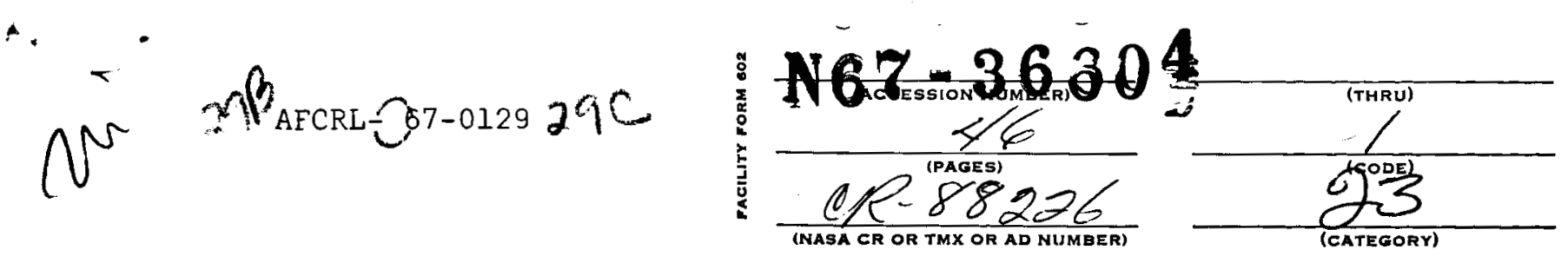

(9) 3 Electromagnetic scattering From Absorbing Spheres 6

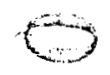

3

Authors

Q)

6 George W. Kattawar and Gilbert N. Plass 9

*..'

Southwest Center for Advanced Studies

Dallas, Texas 3

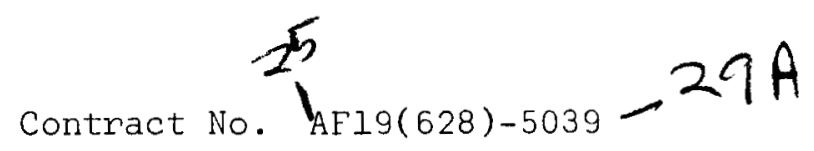

Project No. 4076

Task No. $\quad 407604$

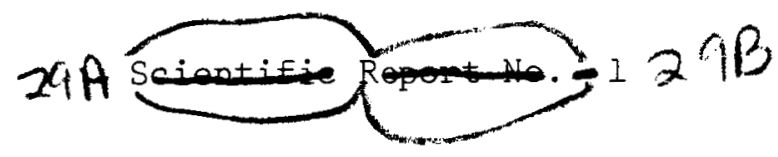

928 December 196610

This research was partially supported by the National Aeronautics and Space Adminsitration

Contract Monitor

Robert W. Fenn

Distribution of this document

is unlimited.

$$
\begin{gathered}
\text { Prepared } \\
\text { for }
\end{gathered}
$$

AIR FORCE CAMBRIDGE RESEARCH LABORATURIES

OFEICE OF AEROSPACE RESEARCH

UNITED STATES AIR. FQRCE

BEDFORD, MASSACHUSETTS 01730 


\title{
ELECTROMAGNETIC SCATTERING FROM ABSORBING SPHERES
}

by

\begin{abstract}
George W. Kattawar* and Gilbert N. Plass
Southwest Center for Advanced Studies Dallas, Texas 75230
\end{abstract}

\section{December 28, 1966}

To be Submitted to Applied Optics

This work was supported by the Ain Force Cambridge Research Laboratories, Office of Aerospace Research contract AF19(628) - 5039 and by the National Aeronautics and Space Administration contract.

*George W. Kattawar is also with North Texas State University, Denton, Texas 76203 . 


\section{ELECTROMAGNETIC SCATTERING FROM ABSORBING SPHERES}

George W. Kattawar and Gilbert N. Plass

Abstract

A very efficient method for the calculation of Mie cross sections for absorbing particles is discussed. It is used to calculate an extensive set of curves which illustrate the dependence of the efficiency factors $\mathrm{Q}$ for absorption, scattering, and extinction on the size parameter $\mathrm{x}$ and on $n_{1}$ and $n_{2}$ (the real and imaginary parts of the index of refraction). Other curves show the height and width of the first resonance in $Q_{\text {ext }}$ as a function of $x$ as well as the average cosine of the scattering angle and the half-width of the angular intensity function as it depends on $\mathrm{x}, \mathrm{n}_{1}$, and $\mathrm{n}_{2}$. Among the interesting features shown are the following: ( 1 ) as $\mathrm{n}_{2}$ increases, $\mathrm{Q}_{\mathrm{sca}}$ decreases to a minimum value before increasirig, when $x \geq 1$ and for most values of $n_{1} ;(2)$ the maximum value of $Q_{\text {ext }}$ at the first resonance decreases rapidly when a small amount of absorption is introduced; (3) over a considerable range of the parameters the width of the first resonance of $Q_{\text {ext }}$ is proportional to $n_{1}{ }^{-4}$ when there is no absorption and to $\mathrm{n}_{2} \mathrm{n}_{1}^{-2}$ when there is absorption; (4) when $\mathrm{n}_{1}$ > 1 , the scattered intensity near the first resonance is predominately forward, symmetrical, or predominately backward when $\mathrm{x}$ is respectively somewhat smaller than, equal to, or larger than the resonance value; (5) as $\mathrm{n}_{2}$ increases, the forward scattered intensity first increases before it decreases, when $x>1$ and for most values of $n_{1} ;(6)$ the half-width of the angular intensity function varies as $\mathrm{x}^{-1}$ when $\mathrm{x} \geq 10$ and is relatively insensitive to the value of $n_{1}$ and $n_{2}$.

George W. Kattawar is with North Texas State University, Denton, Texas, 76203 and the Southwest Center for Advanced Studies, P.0. Box 30365, Dallas, Texas 75230 .

Gilbert N. Plass is with the Southwest Center for Advanced Studies, P.O. Box 30365, Dallas, Texas 75230. 
Introduction

The interaction of an electromagnetic wave with an absorbing sphere is described by the Mie theory and is discussed in detail in Van de Hulst's classic bookl. Unfortunately this book was published before the widespread use of modern computers. At that time the available results for absorbing spheres consisted of some asymptotic equations and a few isolated numerical results. Since the publication of the book, a large number of laboratories have developed computational programs applicable to the case of an absorbing sphere. However, the majority of these investigations have been concerned with a particular substance, such as water droplets (the literature on this subject has been reviewed by Deirmendjian et $a l^{2}$ ). Although these calculations for particular substances are most interesting, it is difficult to gain an understanding of the behavior of the cross sections for absorbing particles from results for isolated values of the index of refraction. Aside from the general discussion in Van de Hulst's book ${ }^{1}$, Deirmendjian et $\underline{a l}^{2}$, Brockes ${ }^{3}$ and Plass ${ }^{4}$ seem to have made the only attempts at understanding the general behavior of these cross sections.

First we present our computational scheme for the calculation of Mie cross-sections of absorbing particles. This scheme is valid for all values of the size parameter which occur in the theory regardless of whether they are very small or very large. Our scheme is both very stable numerically and is easy to program. We believe that it contains improvements over previously reported procedures. 
Next, some new studies are presented of the variation of the Mie cross-sections as a functions of $\mathrm{n}_{2}$, the complex part of the index of refraction. The efficiency factors for absorption, scattering, and extinction are shown as well as the average value of the scattering angle over a wide range of the parameters.

The efficiency factor for extinction has a resonance whose maximum value increases and whose half-width decreases as $n_{1}$, the real part of the index of refraction, increases. The variation of these quantities with both $\mathrm{n}_{1}$ and $\mathrm{n}_{2}$ is discussed.

The scattered intensity as a function of scattering angle decreases from its value of $0^{\circ}$ more and more rapidly as the size parameter increases. This effect is discussed together with its dependence on both $n_{1}$ and $n_{2}$.

Computational Scheme

A computational scheme is presented in this section for the calculation of the Mie cross-sections. It is based on the Ricatti Bessel functions and appears to have some advantages over other schemes which have been described. ${ }^{-8}$ The basic equations can be found in many places in the literature, but their validity should be checked. We shall adopt the notation of Van de Hulst. ${ }^{1}$ The scattering, absorption, and radiation cross-sections can be calculated from various combinations of the sum and products of the coefficients $a_{n}$ and $b_{n} *$. The usual expressions for $a_{n}$ and $b_{n}$ are (reference $1, p .123$ )

*See reference 1, p. 127-128. It should be noted that the expression for $\overline{\cos \theta} Q_{s c a}$ is incorrect as printed. The quantities Re $\left(a_{n} a_{n+1}+b_{n} b_{n+1}\right)$ and $\operatorname{Re}\left(a_{n} b_{n}\right)$ should be replaced by $\operatorname{Re}\left(a_{n} a_{n+1}{ }^{*}+b_{n} b_{n+1}{ }^{*}\right)$ and $\operatorname{Re}\left(a_{n} b_{n}^{*}\right)$. 


$$
\begin{aligned}
& a_{n}=\frac{\psi_{n}^{\prime}(y) \psi_{n}(x)-m \psi_{n}(y) \psi_{n}^{\prime}(x)}{\psi_{n}^{\prime}(y) \zeta_{n}(x)-m \psi_{n}(y) \zeta_{n}^{\prime}(x)}, \\
& b_{n}=\frac{m \psi_{n}^{\prime}(y) \psi_{n}(x)-\psi_{n}(y) \psi_{n}^{\prime}(x)}{m \psi_{n}^{\prime}(y) \zeta_{n}(x)-\psi_{n}(y) \zeta_{n}^{\prime}(x)},
\end{aligned}
$$

where $\mathrm{m}$ is the complex index of refraction, $\mathrm{n}$ is a positive integer, and $\psi$ and $\zeta$ are the Ricatti Bessel functions defined by

$$
\begin{aligned}
& \psi_{n}(z)=z j_{n}(z)=\left(\frac{1}{2} \pi z\right)^{\frac{1}{2}} J_{n+\frac{1}{z}}(z), \\
& \zeta_{n}(z)=z h_{n}(2)(z)=\left(\frac{1}{2} \pi z\right)^{\frac{1}{2}} H_{n+\frac{1}{2}}(2)(z),
\end{aligned}
$$

with $\mathrm{J}_{\mathrm{n}+\frac{1}{2}}$ and $\mathrm{H}_{\mathrm{n}+\frac{1}{2}}(2)$ the Bessel functions of first and third kind and $j_{n}$ and $h_{n}$ the corresponding spherical Bessel functions. The prime denotes differentiation with respect to the argument of the function and

$$
\begin{aligned}
& x=2 \pi a / \lambda, \\
& y=m x
\end{aligned}
$$

where $a$ is the particle radius and $\lambda$ is the wavelength.

These expressions can be written in a much more convenient form for computational purposes, as was first discovered by Aden. ${ }^{4}$ Introduce the logarithmic derivative of the Ricatti Bessel functions,

$$
\begin{aligned}
& D_{n}(y)=\left[\ln \psi_{n}(y)\right]^{\prime}, \\
& G_{n}(x)=\left[\ln \zeta_{n}(x)\right]^{\prime} .
\end{aligned}
$$


Then Eqs. (1) and (2) may be rewritten after some elementary algebraic manipulation in the form

$$
\begin{aligned}
& a_{n}=\frac{\psi_{n}(x)}{\zeta_{n}(x)}\left[\frac{D_{n}(y)-m D_{n}(x)}{D_{n}(y)-m G_{n}(x)}\right], \\
& b_{n}=\frac{\psi_{n}(x)}{\zeta_{n}(x)}\left[\frac{m D_{n}(y)-D_{n}(x)}{m D_{n}(y)-G_{n}(x)}\right] .
\end{aligned}
$$

This is the most convenient expression for the calculation of the coefficients $a_{n}$ and $b_{n}$. The expression outside the brackets involves only the ratio of Ricatti-Bessel functions of real argument. The brackets themselves depend on only two functions: ( 1 ) $G_{n}(x)$, where the argument is always real; (2) $D_{n}(y)$, where the argument may be complex. The method for the computation of these functions is discussed in the remainder of this section.

The logarithmic derivative of the Ricatti-Bessel function, $D_{n}$, satisfies the recurrence relation

$$
D_{n-1}(z)=\frac{n}{z}-\frac{1}{D_{n}(z)+n z^{-1}} \text {. }
$$

The function $G_{n}(z)$ satisfies an identical recurrence relation. These relations were first derived by Infeld ${ }^{9}$ and have been used by several investigators. However, this relationship has always been used in the past to calculate $\mathrm{D}_{\mathrm{n}}$ by an upward recurrence relation. Unfortunately this becomes numerically unstable when $n>|z|$, a region of particular importance for large values of $\mathrm{x}$. On the other hand the downward recurrence formula for $\mathrm{D}_{\mathrm{n}}$ is always numerically stable and is the one which should be user. 
Let

$$
f_{n}(z)=D_{n}(z)+\varepsilon_{n}(z),
$$

where $f_{n}$ is an approximate numerical value of the true value of $D_{n}$ and $\varepsilon_{n}$ is the error in this value. If the approximate value $f_{n}$ is used in Eq. (ll) in order to compute $f_{n-1}$, then

$$
D_{n-I}+\varepsilon_{n-I}=\frac{n}{z}-\frac{1}{D_{n}(z)+\varepsilon_{n}(z)+n z^{-1}} \text {. }
$$

If $D_{n-1}$ is replaced by its value from $E q$. (II), it is found that

$$
\varepsilon_{n-1}=\frac{\varepsilon_{n}}{\left(D_{n}+n z^{-1}\right)\left(D_{n}+\varepsilon_{n}+n z^{-1}\right)} .
$$

When $n>>|z|$, it follows from the series expansion of $D_{n}$ that

$$
D_{n}(z) \cong(n+1) z^{-1}
$$

Thus,

$$
\left|\varepsilon_{n-1}\right| \cong\left|\varepsilon_{n}\right| /\left|(2 n+1) z^{-1}\right|^{2}
$$

or

$$
\left|\varepsilon_{n-1}\right|<<\left|\varepsilon_{n}\right|
$$

Thus the computational error decreases at each step if $D_{n}(z)$ is calculated from Eq. (Il) by downward recurrence in the order $\mathrm{n}$ and the calculation is started at some value $n>>|z|$. The calculations are insensitive to the assumed starting value and rapidly converge to the correct value. In practice zero is a convenient starting value for $D_{n}$. 
In the regions where $n<|z|$, the function $D_{n}$ becomes oscillatory and hence there is no problem in the use of the recurrence relations in this region.

Although the function $G_{n}$ always has a real argument, the value of the function is complex. A similar analysis to that given above shows that an upward recurrence process may always be used with these functions. The function $G_{0}(x)=-i$ for all $x$. Thus the recurrence is always started with this remarkably simple value.

The ratio $\psi_{\mathrm{n}}(\mathrm{x}) / \zeta_{\mathrm{n}}(\mathrm{x})$ in Eqs. (9) and (10) can be expressed in terms of $J_{n}(x)$ and $Y_{n}(x)$, the Bessel functions of first and second kind. These functions are computed from their well-known recurrence relations. $J_{n}$ is computed by downward recursion and $Y_{n}$ by upward recurrence, as has been discussed by several authors. 6,8

A numerically satisfactory method has been presented in this section for the computation of all the functions which occur in Eqs. (9) and (10). This method has been tested in over a thousand cases covering a complete range of values of $\mathrm{x}$ and $\mathrm{m}$. The results agreed in all cases with previously published results and various asymptotic equations where applicable. All calculations were performed on an IBM Model 360-50 computer using double precision arithmetic.

Cross Sections

The complex index of refraction $m$ is the physical parameter which describes the interaction of an electromagnetic wave with an absorbing 
particle. Let us write $m=n_{1}-i n_{2}$. The absorption and scattering cross sections vary with the absorbing power of the particle, which is represented mathematically by the imaginary part of the index of refraction. The variation of the cross sections as a function of $n_{2}$ has not been extensively investigated before the availability of electronic computers, because of the computational difficulties involved. Deirmendjian et al, ${ }^{2}$ Brockes, ${ }^{3}$ and Plass ${ }^{4}$ have given results for particular values of $n_{2}$. Further results are given in this section which more completely illustrate the dependence of these cross sections on $n_{2}$.

In Figs. 1-3, the efficiency factor for absorption $Q_{\text {abs }}$ (absorption cross section divided by cross-sectional area of the particle) is shown as a function of $n_{2}$ for various particular values of $n_{1}$ and $x$. In $F i g .1$, where $x=0.1$, the limiting equation

$$
Q_{a b s}=(\text { constant }) n_{2} \text {, }
$$

is found to be valid when $n_{2} 1$. The curves do not depend markedly on $n_{1}$ until $n_{1} \geq 2$. The value of $Q_{a b s}$ decreases as $n_{1}$ increases for $n_{2}<1$. For the intermediate value $x=1$, it is seen from Fig. 2 that Eq. (1) is valid for $n_{2}<0.1$. The curves are very insensitive to the value of $\mathrm{n}_{1}$ except near the maximum. The value of $\mathrm{Q}_{a b s}$ first increases slightly, and then decreases, as $\mathrm{n}_{1}$ increases, when $\mathrm{n}_{2}<1$.

When $x=10$, Fig. 3 shows that Eq. (18) is only valid for $n_{2}<0.01$. The maximum of the curves is broader than before. However, the curves are still relatively insensitive to the value of $n_{l}$ except near the maximum. 


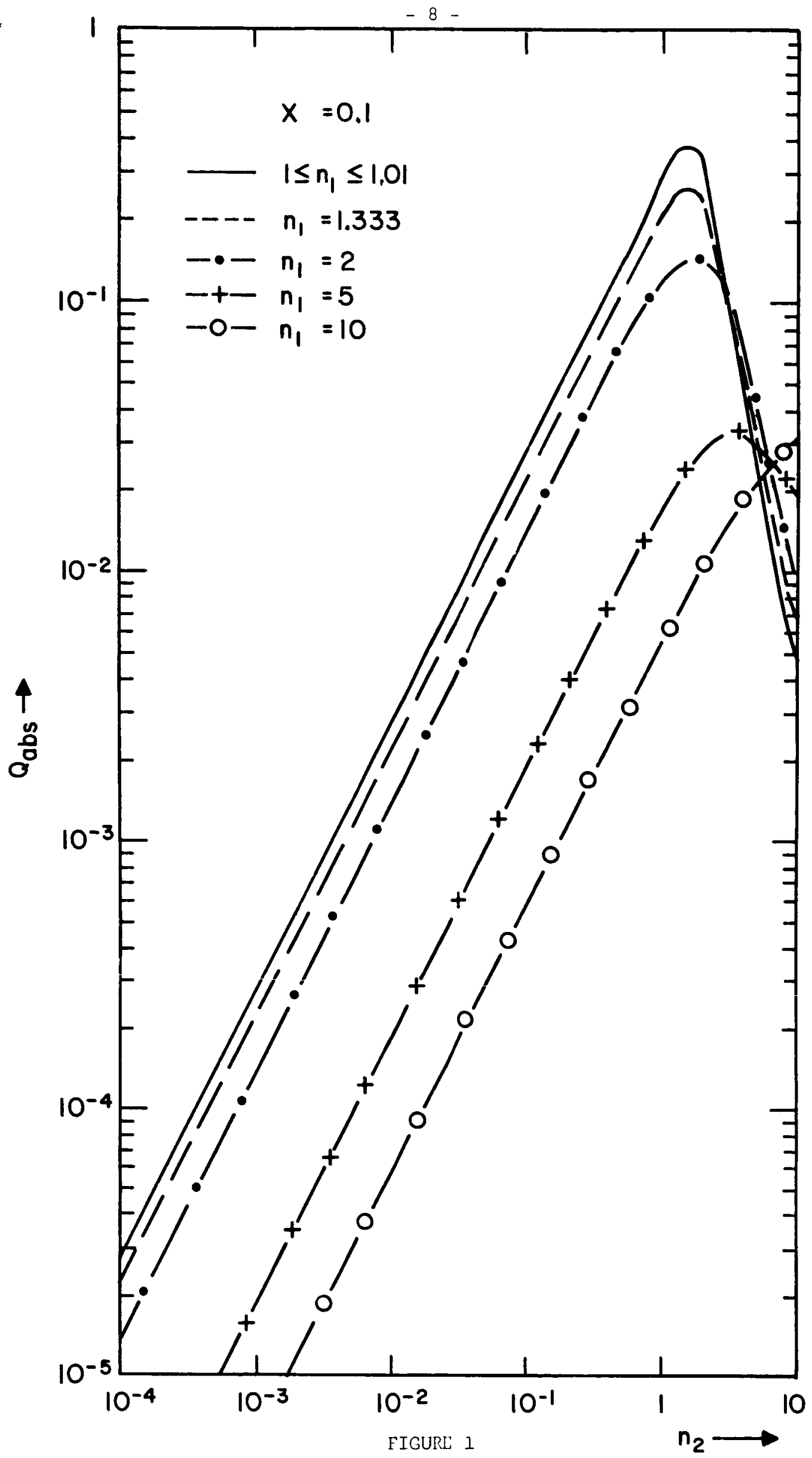




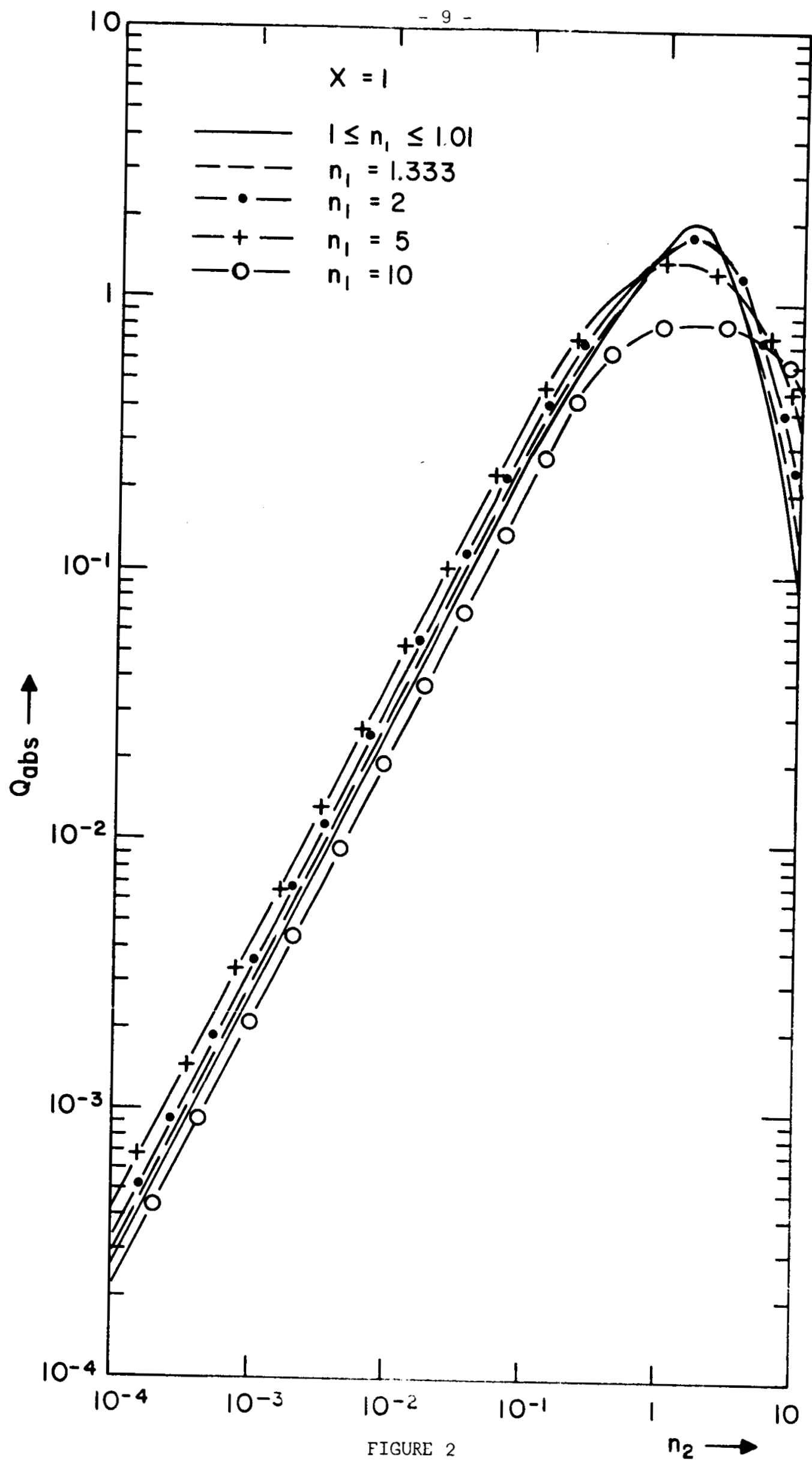




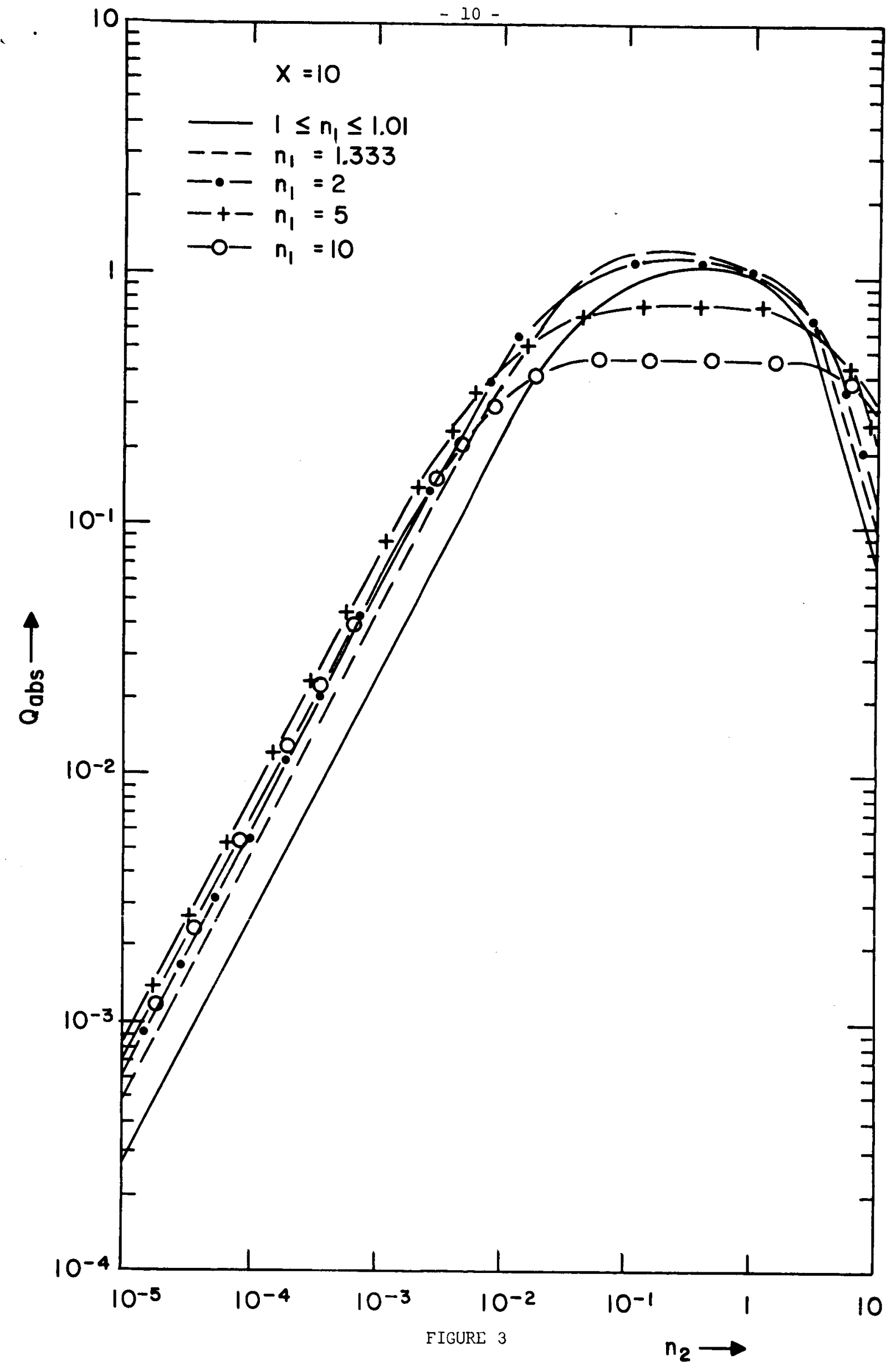


The value of $\mathrm{Q}_{a b s}$ first increases, and then decreases, as $\mathrm{n}_{1}$ increases, when $\mathrm{n}_{2}<0.01$.

The efficiency factor for scattering, $Q_{\text {sca }}$, is shown in Figs. 4-6. $Q_{\text {sca }}$ is independent of $n_{2}$ until $n_{2}$ exceeds some critical value. As would be expected the absorption properties of a sphere have no influence on the scattering until they are sufficiently large. When $x=0.1$, it is seen from Fig. 4 that $Q_{s c a}$ is independent on $n_{2}$ when $n_{2}<0.01$ provided $n_{1} \geq 1.333$. For very small values of $n_{1}$, such as $n_{1}=1.001$, it is found that $Q_{s c a}$ is proportional to $\mathrm{n}_{2}{ }^{2}$ over a region between the point where $Q_{\text {sca }}$ first begins to increase and the maximum of the curve. When $n_{1} \geq 5$, the curves show very little variation with $n_{2}$ over the range calculated. For small values of $x$, the value of $Q_{\text {sca }}$ always increases as $n_{2}$ increases until the maximum of the curve is reached.

When $x=1$, Fig. 5 shows that $Q_{\text {sca }}$ is not influenced appreciably by $\mathrm{n}_{2}$ when $\mathrm{n}_{2} \leq 10^{-2}$. However, a new feature appears in these curves. When $n_{1} \geq 2$, the value of $Q_{\text {sca }}$ first decreases as $n_{2}$ increases. It passes through a minimum value before finally increasing.

For a large value of $x$, such as $x+10$ as shown in Fig. 6, the scattering may be influenced by the absorption of the particle unless $n_{2} \leq 10^{-4}$. When $n_{1} \geq 1.33$, a striking feature of these curves is the very considerable decrease in $Q_{s c a}$ as $n_{2}$ increases. A minimum is reached around $\mathrm{n}_{2}=0.1$ and then $\mathrm{Q}_{\text {sca }}$ increases again. A maximum in the curve may occur beyond $\mathrm{n}_{2}=1$. 


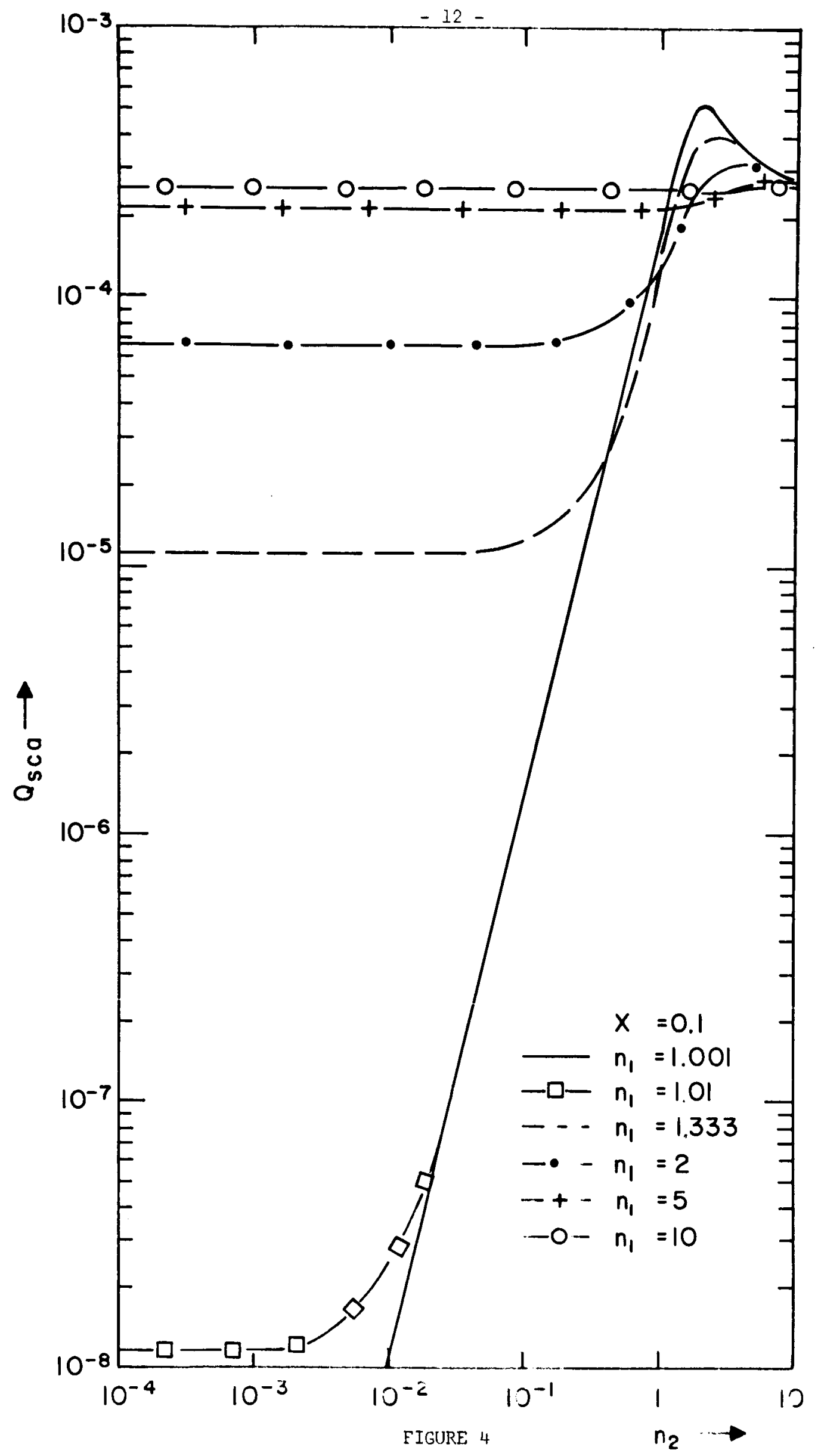




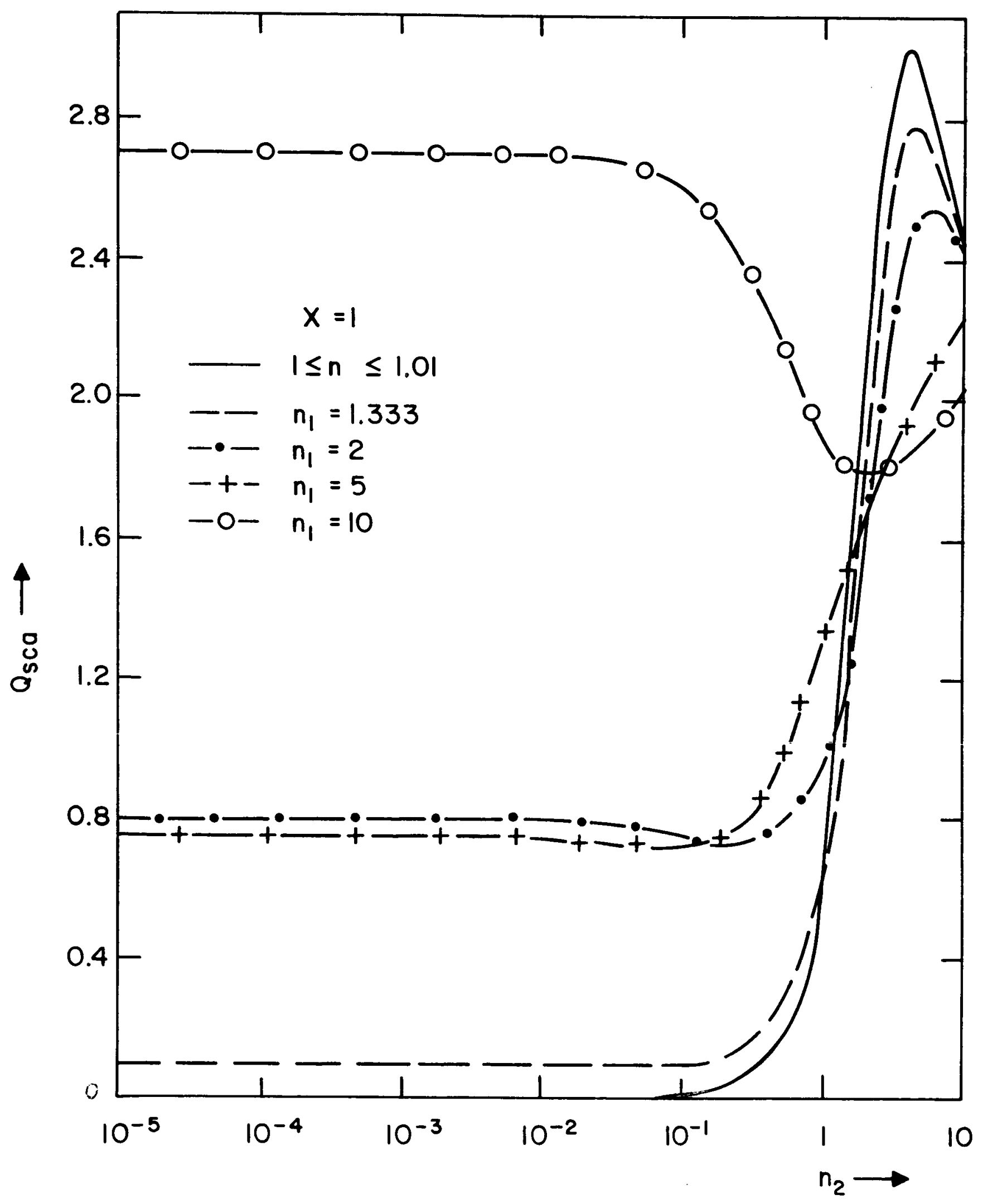

FIGURE 5 


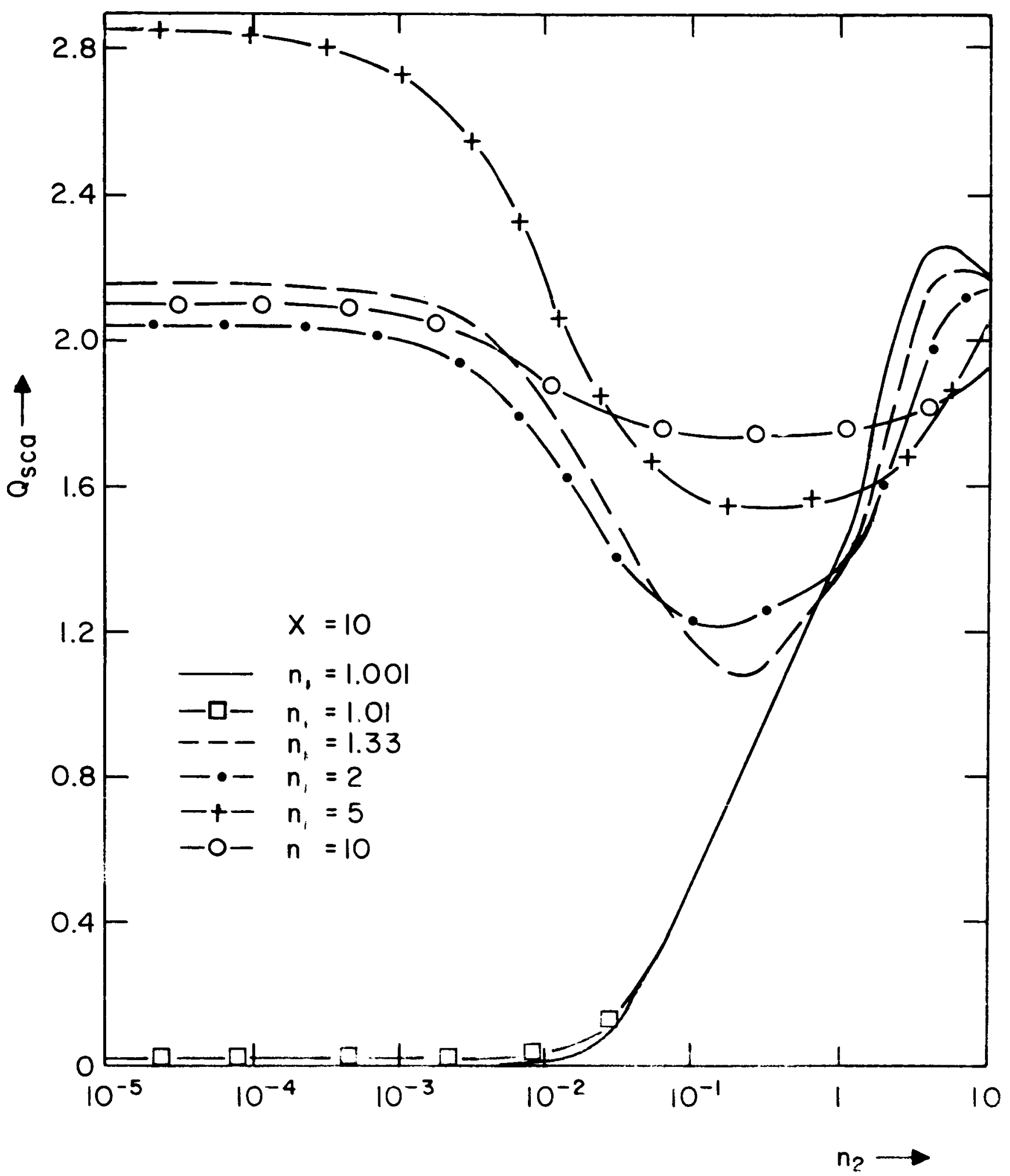


First Resonance of Extinction Cross Section

The curve of the efficiency factor for extinction as a function of the parameter $x$ first increases from zero to a maximum value at $x_{\max }=2.05\left(n_{1}-1\right)^{-1}$, when $n_{1}<1.5$ and the particles are non-absorbing ${ }^{1}$. It is known that this first resonance becomes very narrow as $n_{1}$ increases; at the same time the value of $\mathrm{Q}_{\text {ext }}$ at the maximum Decomes very large. The following approximate formulas are valid when $n_{1}$ is largel.

$$
x_{\max }=\pi n_{1}-1
$$

and

$$
Q_{\text {ext, max }}=6 \pi^{-2} \mathrm{n}_{1}^{2}
$$

where $x_{\text {max }}$ and $Q_{\text {ext, max }}$ are the values of $x$ and $Q_{\text {ext }}$ at the maximum. The above approximate equations are valid only for real values of $\mathrm{m}$. The behavior of this resonance when the particles are absorbing has not been reported, except for the case $n_{1}=50$ (Fig. 18, reference 3 ). The present code was used to calculate the values at the miximum; a special code was developed which calculated the total width of the resonance at half maximum.

The calculated values of $\mathrm{x}_{\max }$ are compared in Table $\mathrm{I}$ with those obtained from the approximate Eq. (19). The values obtained from Eq. (I9) are always too high, but approach the correct value more closely as $n_{1}$ increases. The position of the maximum is amazingly insensitive to the value of $\mathrm{n}_{2}$; the value of $\mathrm{x}_{\max }$ increases slightly as $\mathrm{n}_{2}$ increases. 
Table I. Value of $\mathrm{x}$ at first resonance

\begin{tabular}{|c|c|c|c|}
\hline$n_{1}$ & $\mathrm{n}_{2}$ & $\mathrm{x}_{\max }$ & $x_{\max }$ \\
\hline & & $\begin{array}{l}\text { calculated from } \\
\text { exact equations }\end{array}$ & from Eq. (19) \\
\hline 5 & 0 & 0.6106 & 0.6283 \\
\hline 5 & 0.1 & .6109 & \\
\hline 5 & 1 & .6279 & \\
\hline 10 & 0 & .3113 & .3142 \\
\hline 10 & 0.1 & .3114 & \\
\hline 10 & 1 & .3115 & \\
\hline 20 & 0 & .156696 & .157080 \\
\hline 20 & 0.1 & .156706 & \\
\hline 20 & 1 & .156725 & \\
\hline 50 & 0 & .062807 & .062832 \\
\hline 50 & 0.1 & .062808 & \\
\hline 50 & 1 & .062819 & \\
\hline 70 & 0 & .044871 & .044880 \\
\hline 70 & 0.1 & .044871 & \\
\hline 70 & 1 & .044877 & \\
\hline 100 & 0 & .031413 & .031416 \\
\hline 100 & 0.1 & .031413 & \\
\hline 100 & 1 & .031414 & \\
\hline
\end{tabular}


The value of $Q_{\text {ext }}$ at the first maximum is shown in Fig. 7 as a function of $\mathrm{nl}_{\mathrm{l}}$. The approximate $\mathrm{Eq} \cdot(20)$ is quite accurate for nonabsorbing particles when $n_{1}>10$. The maximum value of $Q_{\text {ext }}$ increases as $n_{1}{ }^{2}$. However, the value at this resonance is greatly reduced when the particles are even slightly absorbing. For a particular value of $\mathrm{n}_{2}$, the value of $\mathrm{Q}_{\text {ext }}$ at the first resonance approaches a limiting value as $n_{1}$ increases.

The total width of the first resonance at half maximum was calculated by a computer routine which searched for the $x$ values on both sides of the resonance which had $Q_{\text {ext }}$ equal to one-half the value at the maximum. This resonance becomes very sharp as $n_{1}$ increases and the half-width becomes correspondingly small. The width at half-maximum is shown in Fig. 8 as a function of $n_{1}$. When $n_{2}=0$, the half-width decreases as $n_{1}{ }^{-4}$. When $n_{1}$ a 100, the half-width is $2.1(10)^{-7}$ compared to the value of $x$ at the resonance of $3.14(10)^{-2}$. On the other hand the half-width is much larger when the particle has any appreciable absorption. As shown in Fig. 8, there is a region in the upper right part of the diagram where the half-width for absorbing spheres is proportional to $\mathrm{n}_{2}$ and $\mathrm{n}_{1}{ }^{-2}$. The ability of a relatively small amount of absorption both to broaden the resonance and to reduce its peak value is very striking.

Influence of Absorption on Angular Distribution

The variation of the angular distribution for the scattered intensity has been given by $\mathrm{Plass}^{4}$ for some particular cases over a wide range of 


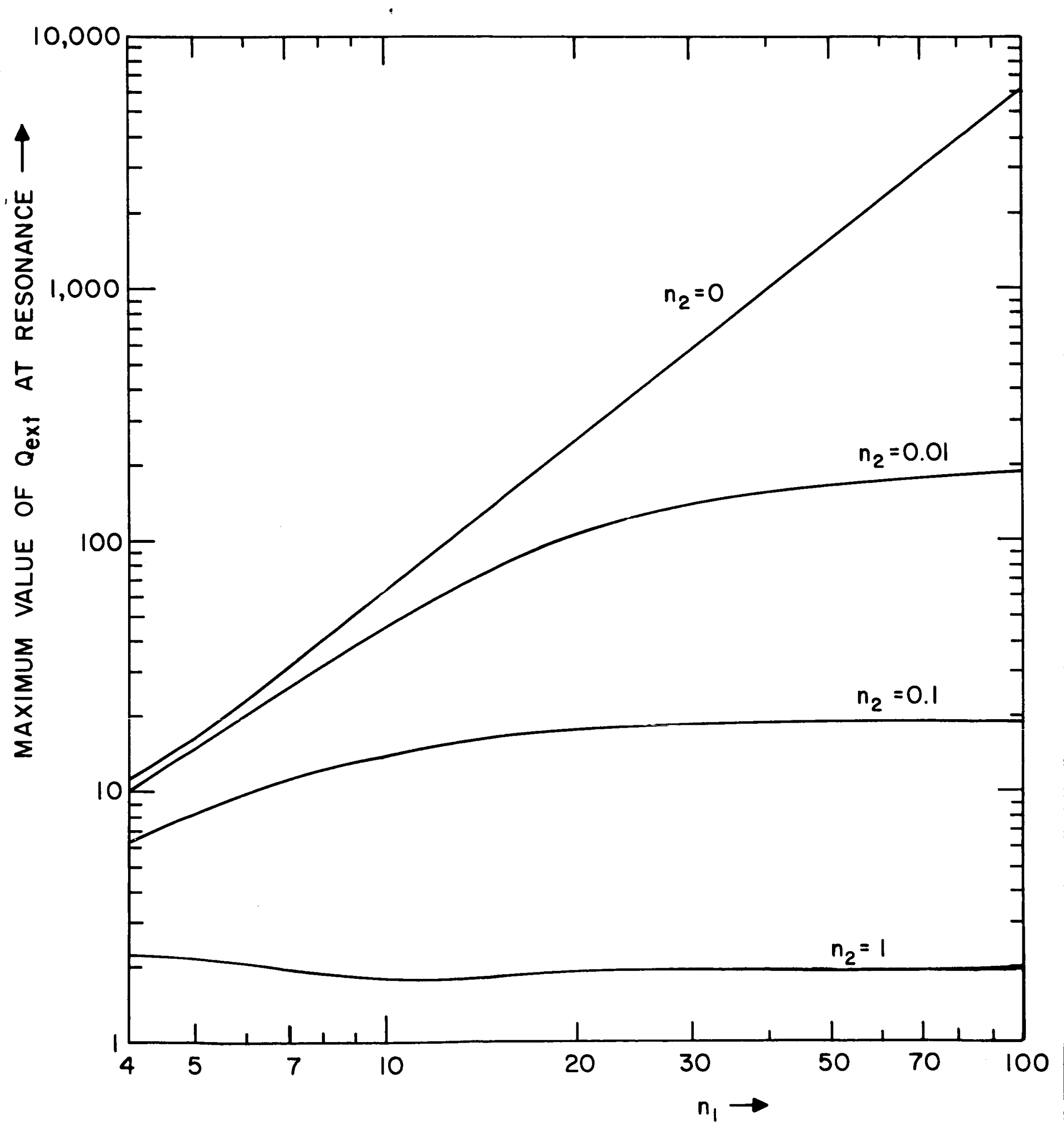

FIGURE 7 


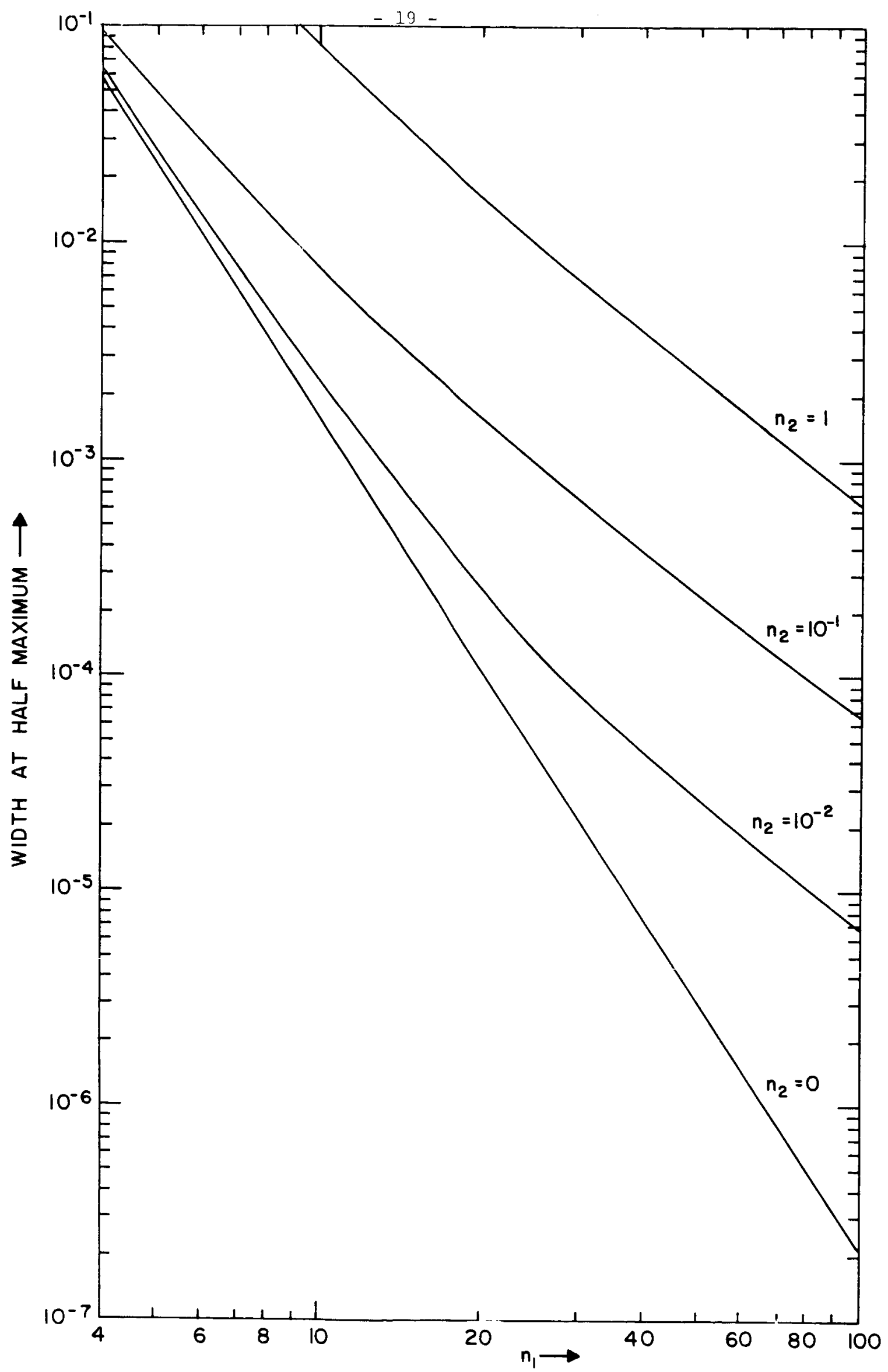

FIGURE 8 
values of $n_{2}$ when $x=1,5$, and 8 . The average value of the cosine of the scatterirg argle over botr directions of polarization (as defined by Van de Hulst, p. I28) contains a considerable amount of information about the predominant scattering directions in a single numenical value. It then becomes possible to present this information in graphical form for a wide range of values of the other parameters of the protlem.

In Figs. 9-1l the average value of the cosine of the scattering angle is given as a function of $\mathrm{n}_{2}$. The curves in Fig. 9 are for $\mathrm{x}=0.1$. For this small value of $x$, the scattering is given closely by the Rayleigh scattering Eunction which is symmetric about $\theta=90^{\circ}$. Eor $\mathrm{n}_{2}<0.1$, the average scattering angle is slightly in the forward direction; the average value of the cosine, $\langle\cos \theta\rangle$ av , is around 0.002 . Thus, $\langle\theta\rangle_{\text {av }}$ is of the order $\frac{1}{2} \pi-0.002$ radians. The angular distribution is not appreciably influenced by the absorption of the spherical particle until $\mathrm{n}_{2}$ is of the order of unity. Then the scattering distribution changes rapidiy as $\mathrm{n}_{2}$ increases still further; the $\langle\cos \theta\rangle_{\text {av }}$ becomes negative, which indicates a slight preponderance of backward scattering when $n_{2}$ is large. For example, $\langle\cos \theta\rangle_{a v}=-0.0237$ when $n_{1}=1.33$ and $n_{2} \square 10$.

The curves in Fig. 10 for $x$ a 1 exhibit a considerably more complex behavion. In the first place the value of $\langle\cos \theta\rangle_{\text {av }}$ in the limit of small absorption depends on the value of $n_{l}$; it is positive when $n_{l} \leq 2$ $\left(\langle\theta\rangle_{a v}=1.29\right.$ radians on $74^{\circ}$ when $\left.n_{l}=2\right)$ and negative when $n_{1}=5$ and 10 $\left(\langle\theta\rangle_{\mathrm{av}}=1.60\right.$ radians on $103^{\circ}$ when $\left.\mathrm{n}_{1}=5\right)$. The angular distribution is 


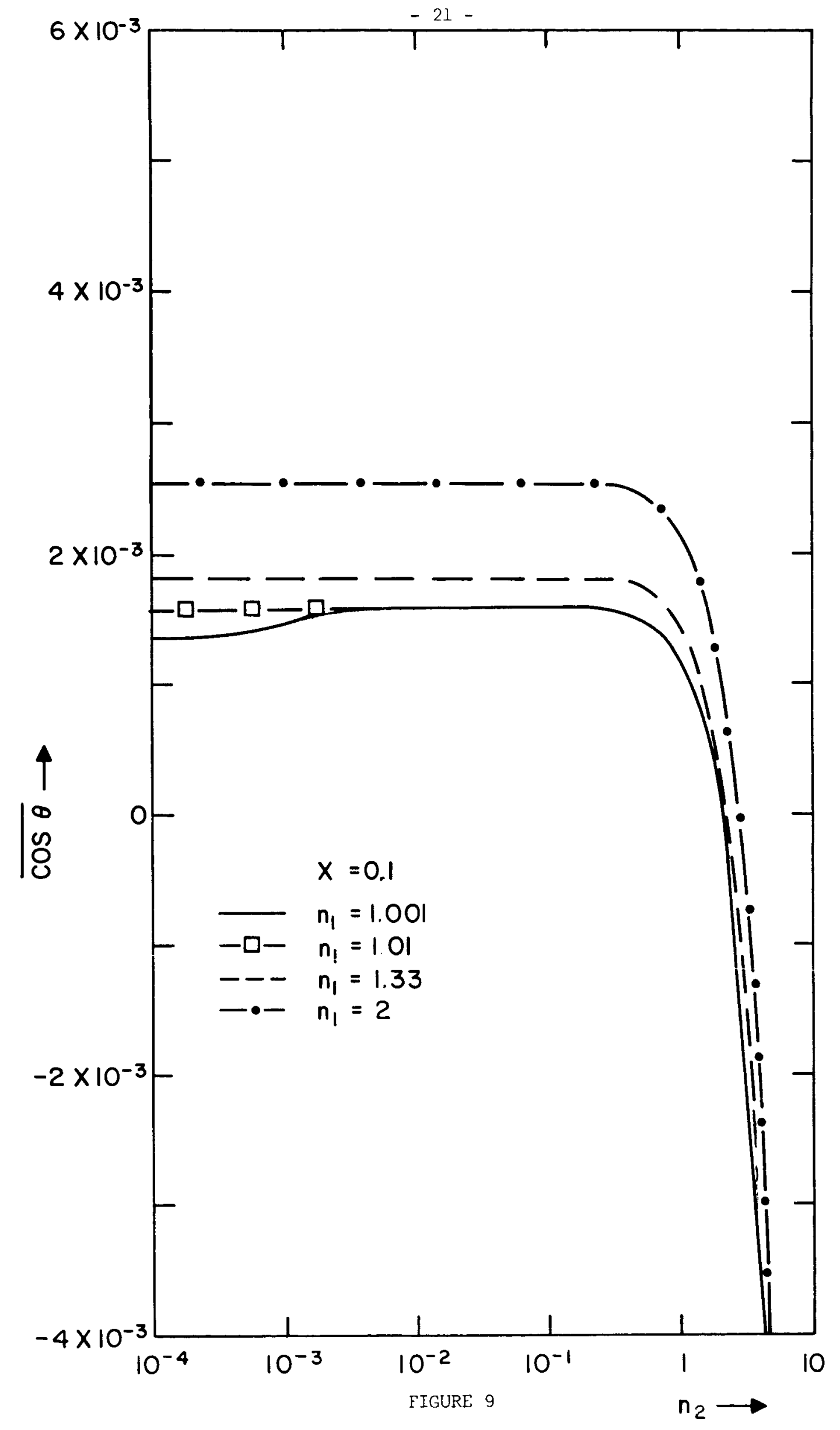




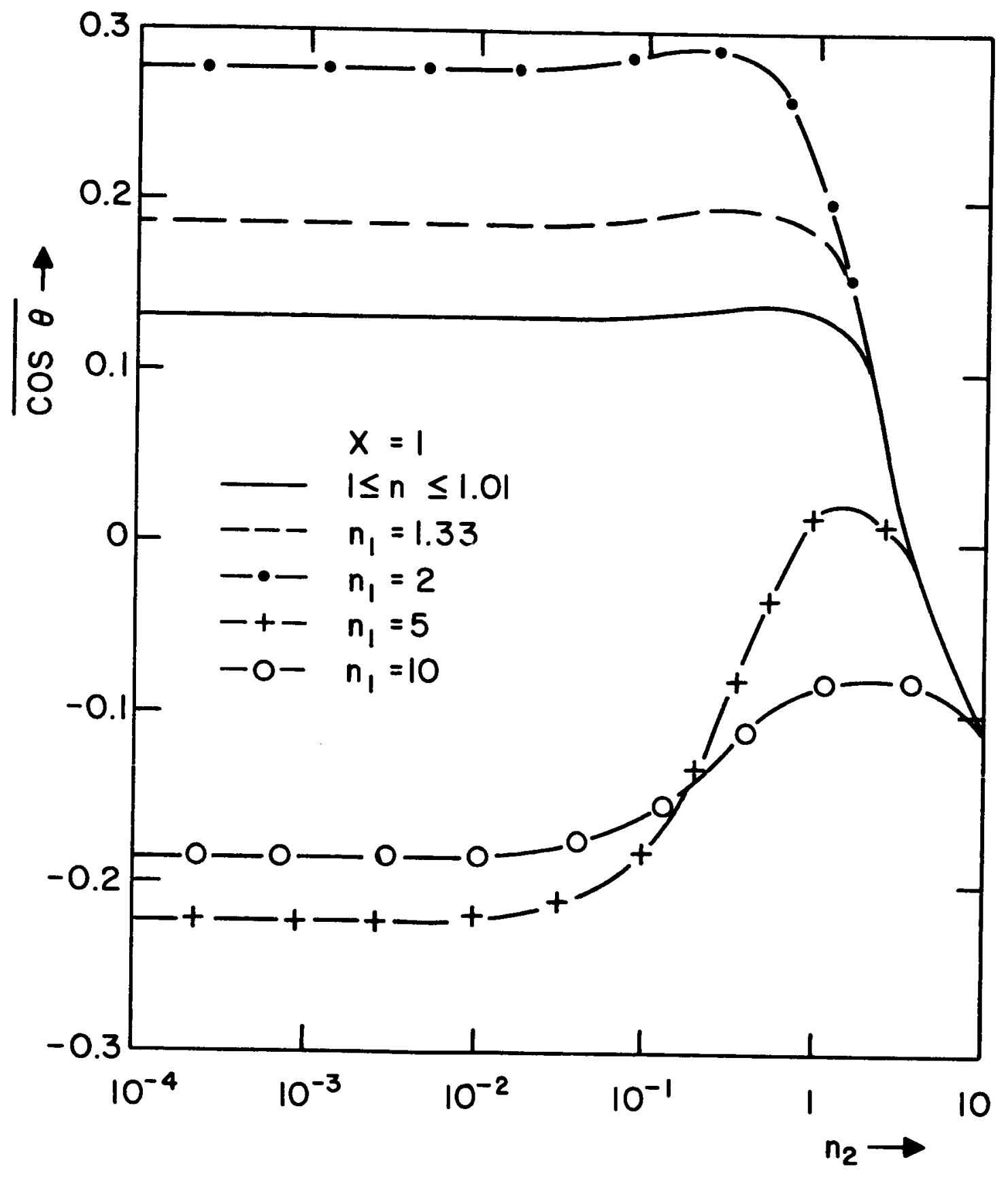




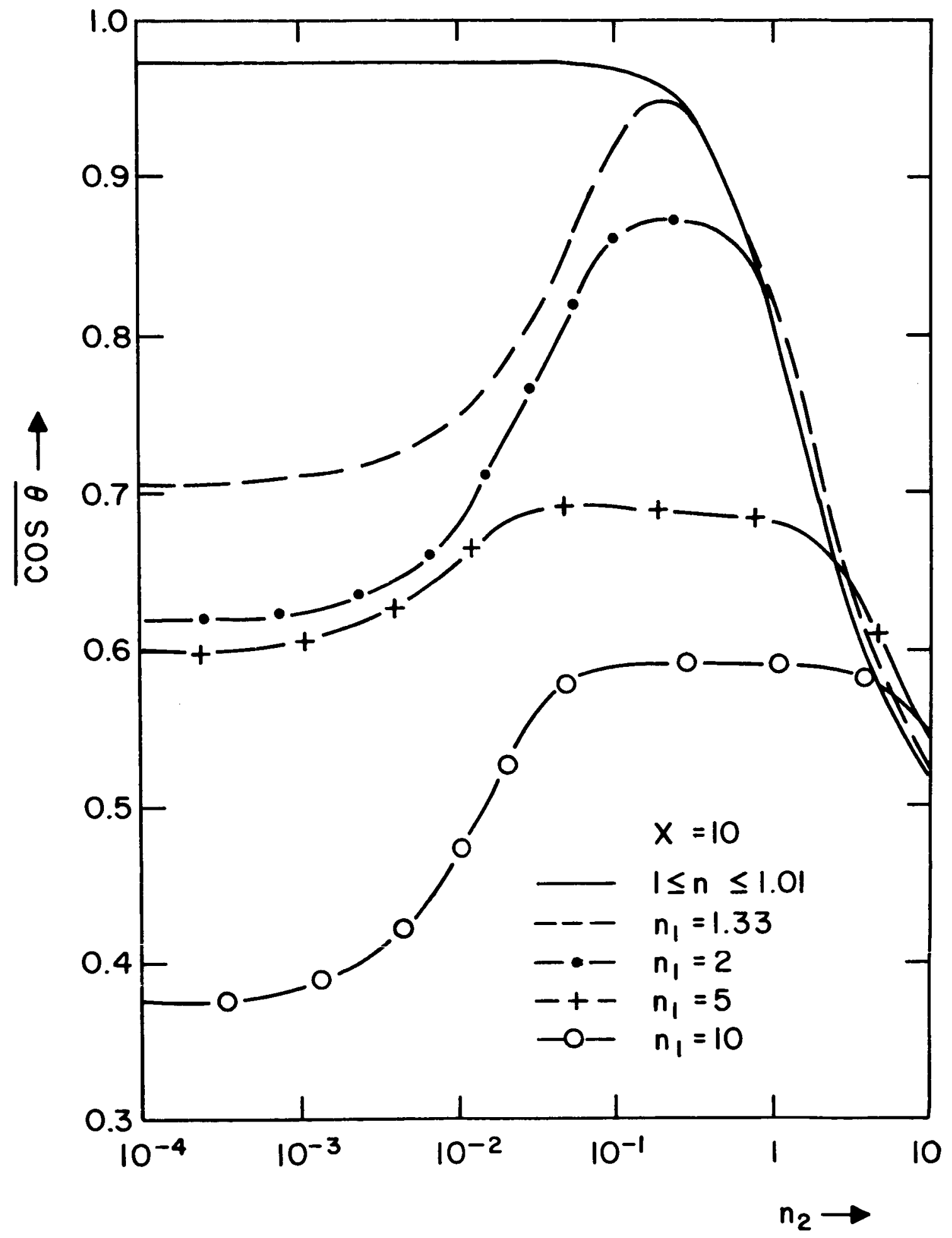


first influenced by $n_{2}$ at a value of approximately $0.01 ;\langle\cos \theta\rangle_{a v}$ increases to a maximum value and then decreases again as $n_{2}$ increases. The value of $\langle\cos \theta\rangle$ av is positive for $n_{1}=5$ over a narrow range near $n_{2}=1$. When $n_{2}>>1,\langle\cos \theta\rangle_{\text {av }}$ is negative. It is interesting to compane these curves for $n_{1}=1.33$ with $\mathrm{Fig}$. 16 of reference 3 .

The curves for $x=10$ are shown in Fig. 11. Fon large values of $x$ the scattering is strongly forward; thus all values of $\langle\cos \theta\rangle$ av shown in this figure are positive. For small values of $n_{2},\langle\theta\rangle_{a v}=0.238$ radians on approximately $14^{\circ}$ when $n_{1} \leq 1.01$. As $n_{2}$ increases all of the curves for $n_{1} \geq 1.33$ pass through a broad maximum dnd then decrease. The maximum forward scattering usuaily occurs for $\mathrm{n}_{2}$ in the range from 0.1 to 1 (compare with Fig. 17 of reference 3 ). Except for very small values of $\mathrm{n}_{l}$, the forward scattering is less pronounced for weakly absorbing spheres. It is instructive to compare these results with the very interesting curves of Irvine. 10

Angular Distribution Near a Resonance

The angular distribution exhibits an anomalous behavior near the resonances of $Q_{\text {ext }}$. This is illustrated in Figs. 12-14 when $n_{1}=10, n_{2}=0$. Fon comparison purposes $Q_{\text {ext }}$ is also plotted in Figs. 12 and 13. The values of $x$ at the resonances shown on these figures are given in Table II together with the maximum value of $\mathrm{Q}_{\text {ext }}$ at each resonance.

For small values of $x$ the scattering might be expected to follow the Rayleigh distribution and to be symetric around $90^{\circ}$. The actual scattering 


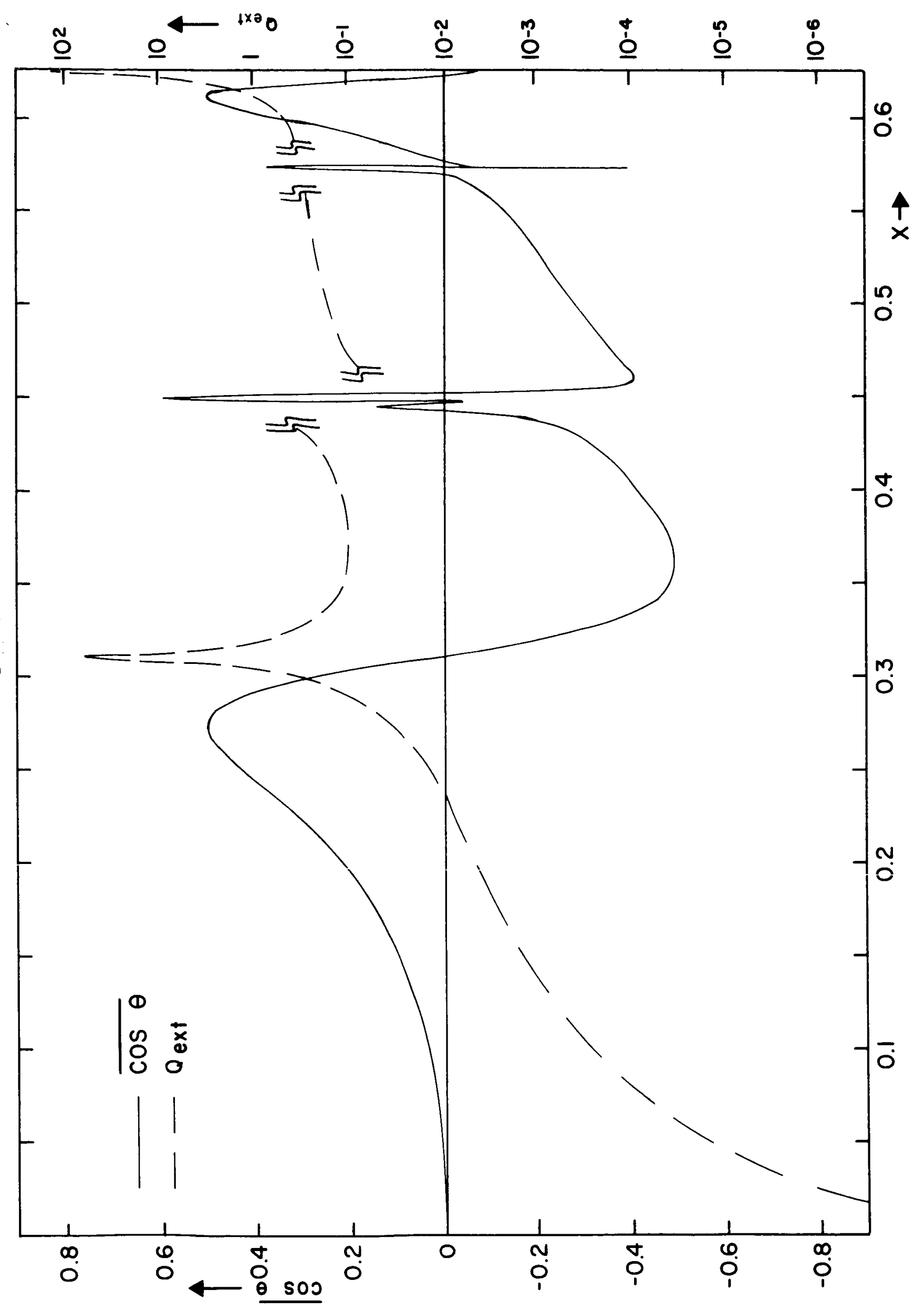




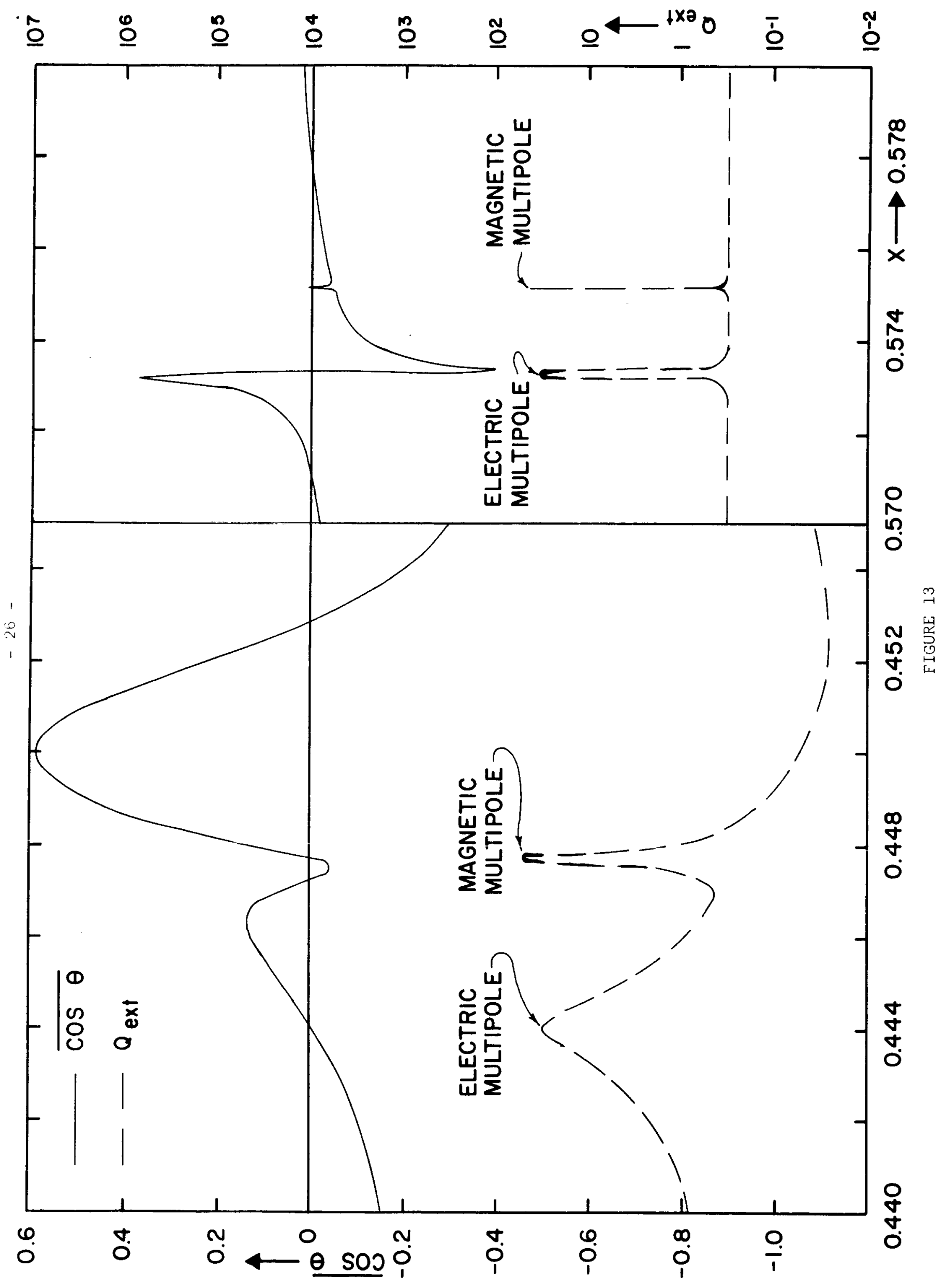



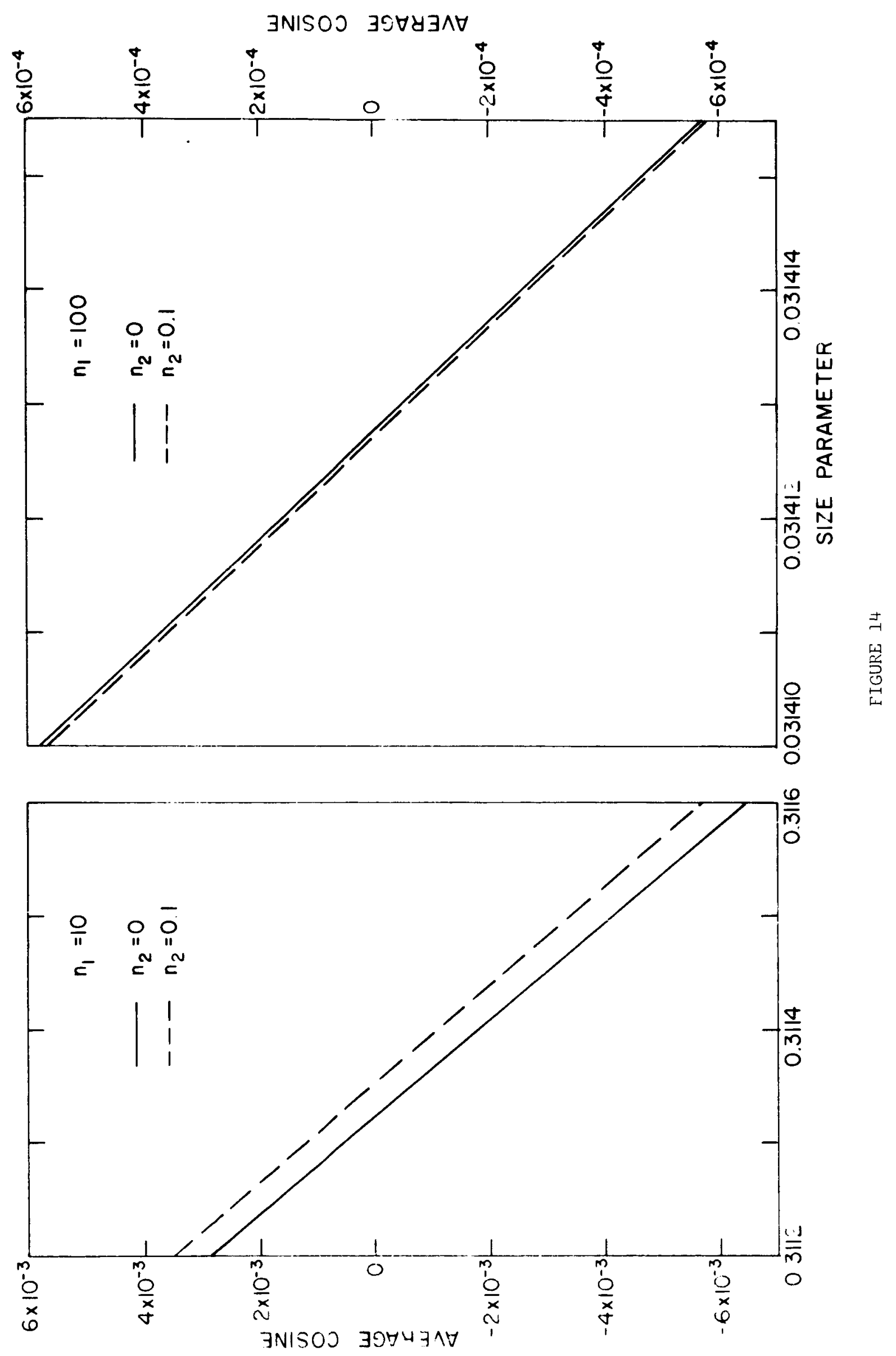
Table II. Value of $x$ and $Q_{\text {ext }}$ at resonance for $n_{1}=10, n_{2}=0$.

Value of $n$

(see Eqs. (1) and (2))

1

1

2

2

3
Type of multipole

magnetic

electric

magnetic

electric

magnetic $\mathrm{x}$

0.31131

0.44404

0.44775

0.57330

0.57516
Qext

61.9

30.5

50.0

30.7

42.3

pattern is very far from this simple distribution. The value of $\langle\cos \theta\rangle_{\mathrm{av}}$ is small when $x<0.1$. However as shown in Fig. 12, as $x$ increases toward the first resonance, $\langle\cos \theta\rangle_{\mathrm{av}}$ increases to a maximum value of 0.50 at $\mathrm{x}=0.275$. Its value then decreases rapidly and passes through zero at nearly the same value of $\mathrm{x}$ at which the resonance occurs. The value of $\langle\cos \theta\rangle_{\mathrm{av}}$ then becomes increasingly negative and reaches a minimum at $x=0.360$ when $\langle\cos \theta\rangle_{a v}=-0.50$.

The same type of behavior occurs at each of the higher multipole resonances as is shown in more detail in Fig. 13. At each resonance $\langle\cos \theta\rangle_{\text {av }}$ passes through zero at nearly the resonance point. For values of $\mathrm{x}$ slightly smallen than the magnetic dipole resonance $(n=1)$, the scattering is predominately forward; for the higher magnetic multipoles, the scattering as influenced by the resonance is predominately backward for $\mathrm{n}=2$ and forward for $\mathrm{n}=3$ (where the interaction is not sufficiently strong to entirely alter the general trend of the curve) when $\mathrm{x}$ is 
slightly smaller than the resonance value. Similarly for the electric multipoles when $\mathrm{x}$ is slightly smaller than the resonance value, the scattering is predominately backward for $\mathrm{n}=1$ and forward for $\mathrm{n}=2$. The influence of the multipole resonance on the scattering oscillates in this manner as each higher resonance is encountered.

The electric and magnetic multipole resonances tend to occur in closely spaced pairs. As the order of the multipole increases these pairs tend to become closer together and the resonances become sharper. The magnitude of $Q_{\text {ext }}$ for both the magnetic and electric multipole resonances are predicted quite closely by the approximate equation given on p. 157 of Van de Hulst ${ }^{1}$.

The value of $\langle\cos \theta\rangle_{\mathrm{av}}$ in the immediate vicinity of the first resonance is shown in Fig. 14 for $n_{1}=10$ and 100 and $n_{2}=0$ and 0.1 . This figure shows in more detail that $\langle\cos \theta\rangle$ av is zero at a value of $x$ that is extremely close to the resonance value for $x$. These curves are only very slightly influenced by the value of $n_{2}$. The values of $\langle\cos \theta\rangle_{\mathrm{av}}$ for $\mathrm{n}_{1}=100$ are the same as those for $\mathrm{n}_{1}=10$ on the scale of Fig. 12 up to $x=0.4$ (except for some very minor variations such as crossing the axis at the first resonance at a slightly larger $x$ value), provided the $\mathrm{x}$ values for $\mathrm{n}_{1}=100$ are multiplied by 10 before comparison.

The angular intensity patterns are so unusual near a resonance that three examples of the complete patterns are given in Fig 15 for $n_{1}=10, n_{2}=0$. The values of $x$ chosen for this graph $(x=0.275,0.31131,0.360)$ correspond to the maximum forward scattering, scattering at the resonance 


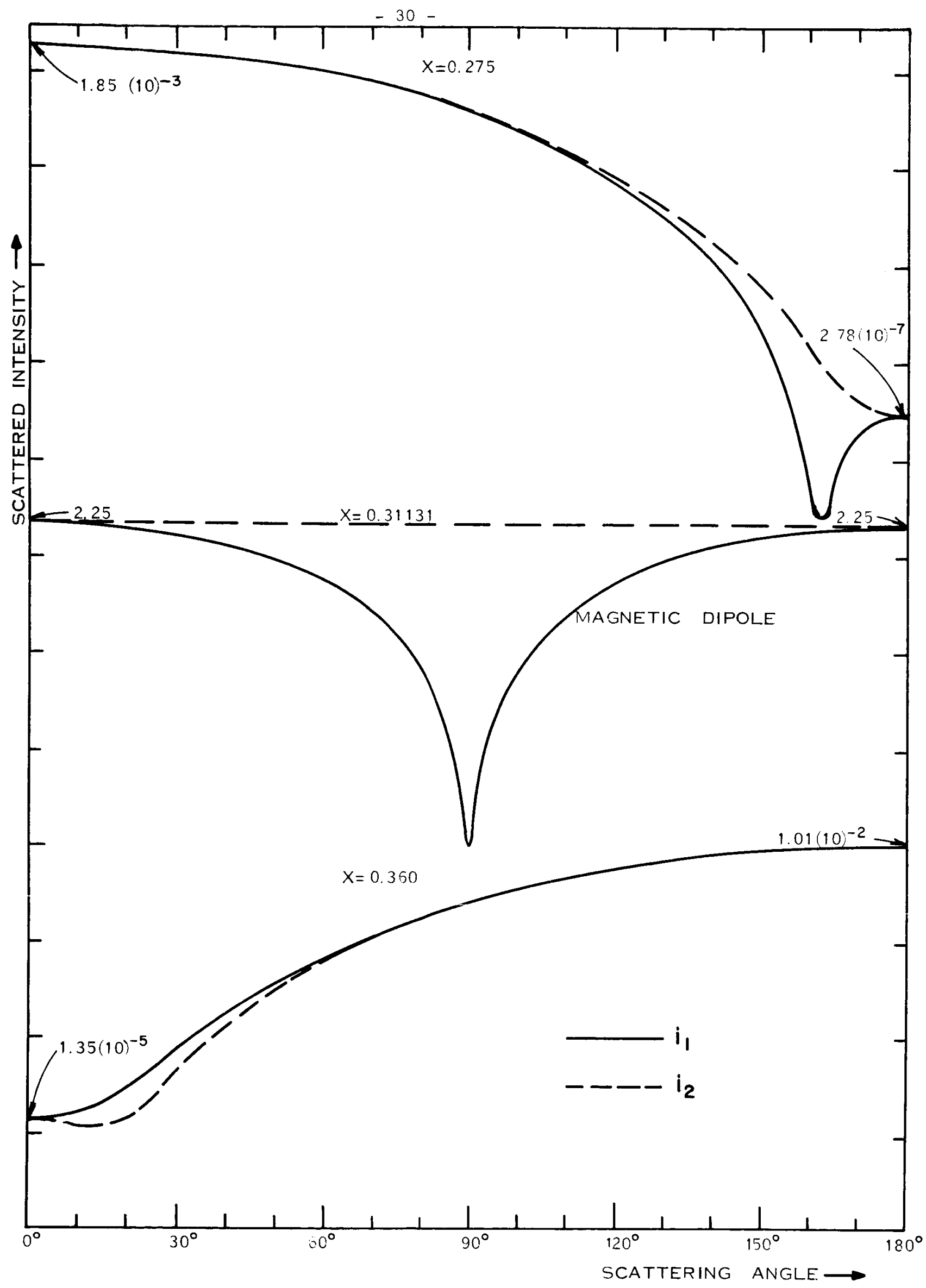

FIGURE 15 
point, and the maximum backward scattering. When $x=0.275$ the intensity functions $i_{1}$ and $i_{2}$ as defined by Van de Hulst ${ }^{1}$ are $1.85 \times 10^{-3}$ in the forward direction $\left(\theta=0^{\circ}\right)$ and $2.78 \times 10^{-7}$ in the backward direction $\left(\theta=180^{\circ}\right)$. At the resonance point for magnetic dipole scattering $\mathrm{x}=0.31131$. The intensity $i_{2}$ is constant and equal to 2.25 to three significant figures at all angles; the intensity $i_{1}$ has a sharp minimum at $90^{\circ}$ and is very nearly symmetrical about $90^{\circ}$. This is the Rayleigh scattering pattern expected for small values of $x$, except that the roles of $i_{1}$ and $i_{2}$ are reversed for magnetic dipole resonance scattering. When $\mathrm{x} \mathbf{0} 0.360$ the pattern has changed to strong backwand scattering with an intensity of $1.35 \times 10^{-5}$ at $\theta=0^{\circ}$ and $1.01 \times 10^{-2}$ at $\theta=180^{\circ}$.

When $x<0.1$ the intensity $i_{1}$ is nearly constant and $i_{2}$ has a sharp minimum near $90^{\circ}$ as expected for Rayleigh scattering. As $x$ increases the angular pattern is distorted by the magnetic dipole resonance as shown in Fig. 15. The higher multipoles similarly change the scattering pattern in a more and more complicated manner as the order of the multipole increases. The oscillations in $\langle\cos \theta\rangle_{\text {av }}$ near the first five multipoles are shown in Figs. 12 and 13. The actual angular distributions near these multipoles are so interesting that they are shown in Fig. 16. The angular distribution at the electric dipole resonance $(x=0.44404)$ is the same as for the magnetic dipole, except the roles of $i_{1}$ and $i_{2}$ are reversed.

The magnetic and electric quadrupole resonances at $\mathrm{x}=0.44775$ and $x=0.57330$ respectively have similar angular intensity functions except for the interchange of $i_{1}$ and $i_{2}$. For the magnetic quadrupole, it is seen from Fig. 16 that the minimum of $i_{2}$ occurs at $90^{\circ}$ and the minima of 


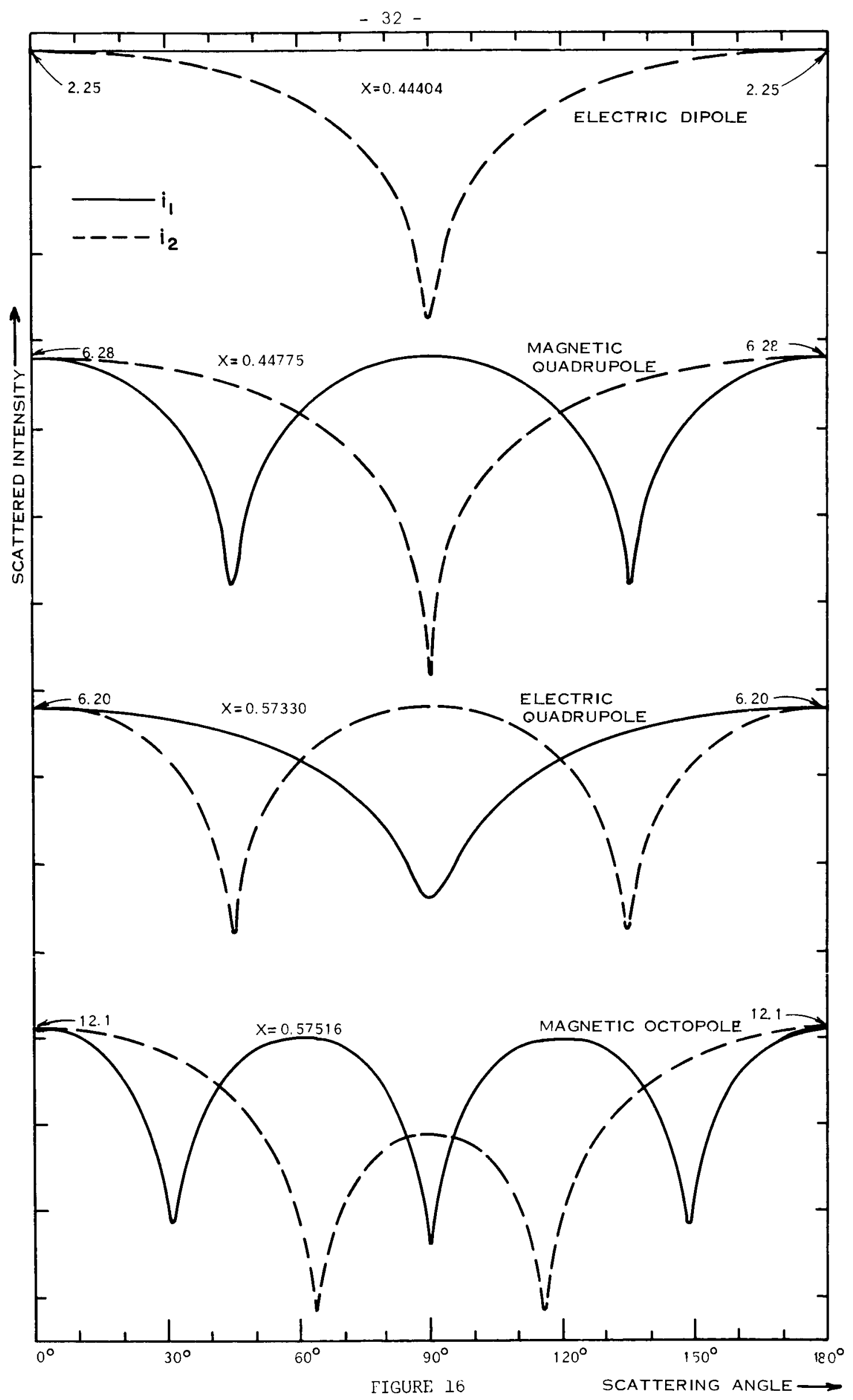


$i_{1}$ occur at $45^{\circ}$ and $135^{\circ}$.

The magnetic octopole resonance at $x=0.57516$ is also shown in Fig. 16 . The following electric octopole resonance has a similar pattern with $i_{1}$ and $i_{2}$ interchanged. Calculation shows that the minima of $i_{1}$ occur at $31^{\circ} 5^{\prime}, 90^{\circ}$, and $148^{\circ} 55^{\prime}$ and those of $i_{2}$ at $63^{\circ} 26^{\prime}$ and $116^{\circ} 34^{\prime}$. All of these curves are very nearly symmetrical around $90^{\circ}$.

The general shape of these resonance scattering curves can readily be explained from the general equations for angular Mie scattering (e.g. p. 125 Van de Hulst $\left.{ }^{1}\right)$. At the magnetic dipole resonance the coefficient $b_{1}$ is much larger than any of the others. It follows from the equations that $i_{1} \propto \cos \theta$ and $i_{2}=$ constant. This angular dependence is slightly modified by the other terms in the series with the result that $i_{1}$ is different from zero at $\theta=90^{\circ}$ and the distribution is very slightly asymmetric around $\theta=90^{\circ}$.

At the magnetic quadrupole resonance, the coefficient $b_{2}$ is dominant and the same equations show that $i_{1} \propto \cos 2 \theta$ and $i_{2} \propto \cos \theta$. The observed pattern as shown in Fig. 16 follows from this dependence. Similarly the coefficient b3 dominates at the magnetic octopole resonance; thus $i_{1} \propto \cos \theta+15 \cos 3 \theta$ and $i_{2} \approx 5 \cos ^{2} \theta-1$. Again the observed angular pattern follows from these functions as slightly modified by the other terms in the summation. At resonance $\langle\cos \theta\rangle$ av would be exactly zero, except for the contribution from other terms in the series. We have already remarked in connection with Fig. 14 that $\langle\cos \theta\rangle$ av is zero for a value of $x$ nearly equal to, but slightly different from the resonance value. 
The electric multipole resonances occur when a coefficient $a_{n}$ is dominant. The fundamental equations show immediately that the angular dependence is the same as for the magnetic multipole resonance for the same value of $\mathrm{n}$ as far as the contribution from the leading terms is concerned, provided the roles of $i_{1}$ and $i_{2}$ are interchanged. The actual curves may be somewhat different quantitatively, because of the contribution from other terms in the series. This can be seen by comparing the curves for the magnetic and electric quadrupole resonance shown in Fig. 16 . The electric quadrupole resonance at $90^{\circ}$ is broader and shallower than the corresponding curve for the magnetic quadrupole.

The angular intensity curves have been discussed in great detail for the case $n_{1}=10$ and $n_{2} \quad 0$, because all of the resonances considered here occur at a value of $x<1$. Thus, the additional effects due to interference between the various reflected and refracted rays which are introduced when $x>1$ are avoided. However, the angular variation at the resonance points is qualititively the same for any non-absorbing sphere.

One can better understand the angular variation for moderate values of $\mathrm{n}_{1}$ by thinking of the superposition of the angular patterns discussed here on the patterns caused by interference between the various reflected and refracted rays. For example, the angular patterns for $n_{1}=2$ are given on $\mathrm{p}$. 152 of Van de Hulst ${ }^{1}$. When $\mathrm{x}=1$, the curves are those expected for Rayleigh scattering. At $x=1.5$, the magnetic dipole resonance at $\mathrm{x}=2.2$ modifies the curves and they look like the upper set in Fig. 15 . 
At $x=2$ and $x=2.5, i_{1}$ is less than $i_{2}$ at almost all angles. Calculation shows that the expected resonance scattering curve (similar to the middle curves in Fig. 15) occurs at the resonance. The additional wiggles in these curves are caused by the superposition of the interference effects mentioned previously on the resonance scattering.

The magnetic dipole resonance occurs at the smallest value of $x$ of any resonance. After this the resonances occur in pairs. The electric multipole resonance of order $\mathrm{n}-1$ always occurs at a slightly smaller value of $\mathrm{x}$ than the magnetic multipole resonance of order $\mathrm{n}$. The halfwidth of the resonances becomes narrower as the order increases and can easily be missed unless very fine intervals are taken in the calculations.

Strong forward scattering occurs on one side of a resonance and strong backward scattering on the other side. This effect may be obscured by other factors for high multipole resonances, but is very prominent for the first few resonances. The scattering is very nearly symmetric around $90^{\circ}$ at the resonance.

Half-width of Angular Intensity Function

The angular intensity functions for the two directions of polarization $i_{1}(\theta)$ and $i_{2}(\theta)$ (as defined by Van de Hulstl) have their maximum values in the forward direction, $\theta=0^{\circ}$, in most cases. The only exception is when $\mathrm{x}$ is small and $\mathrm{n}_{2}$ is large. Whenever $\mathrm{x} \gg 1$, there is a strong maximum in the forward scattering. This maximum becomes stronger and sharper as $x$ increases and is largely due to diffraction effects from the scattering sphere. Thus the half-width of the intensity maximum should 
be proportional to $x^{-1}$ when $x>>1$. The actual variation of this half-width as a function of $x, n_{1}$, and $n_{2}$ has not been reported previously. A computer program was developed which searched for the value of nearest $0^{\circ}$ that resulted in a value of $i_{2}(\theta)$ equal to one-half the value of $i_{2}\left(0^{\circ}\right)$. The functions $i_{1}$ and $i_{2}$ are equal at $\theta=0^{\circ}$ and are nearly equal near the forward direction. The half-widths calculated from either of these functions were identical on the scale of these graphs, except in a few cases when $x \ll 1$. Since we are primarily interested in the half-widths for large values of $\mathrm{x}$, only the results calculated from $i_{2}$ are shown.

In Fig. 17 the half-width in degrees is plotted as a function of $\mathrm{x}$ for $n_{2}=0$ and various values of $n_{1}$. When $x \ll 1$, the half-width is nearly $45^{\circ}$ in all cases. As $x$ increases, the half-width starts to decrease around $x=1$. The half-width is nearly proportional to $x^{-1}$ when $x>>1$; this indicates that the principal cause of the strong forward scattering for this range of $\mathrm{x}$ is diffraction effects by the sphere. There are moderate variations in the curves as $n_{1}$ is varied, but the general similarity of all the curves is a noteworthy feature.

The dependence of the half-width on $\mathrm{n}_{2}$ is shown in Figs. 18 and 19 . Again the curves show that the half-width is nearly proportional to $\mathrm{x}^{-1}$ whenever $x>>1$. The changes in the half-width as $n_{2}$ is varied between zero and unity are surprisingly small. From these results it is possible to estimate the extent of the forward scattering with considerable precision. 


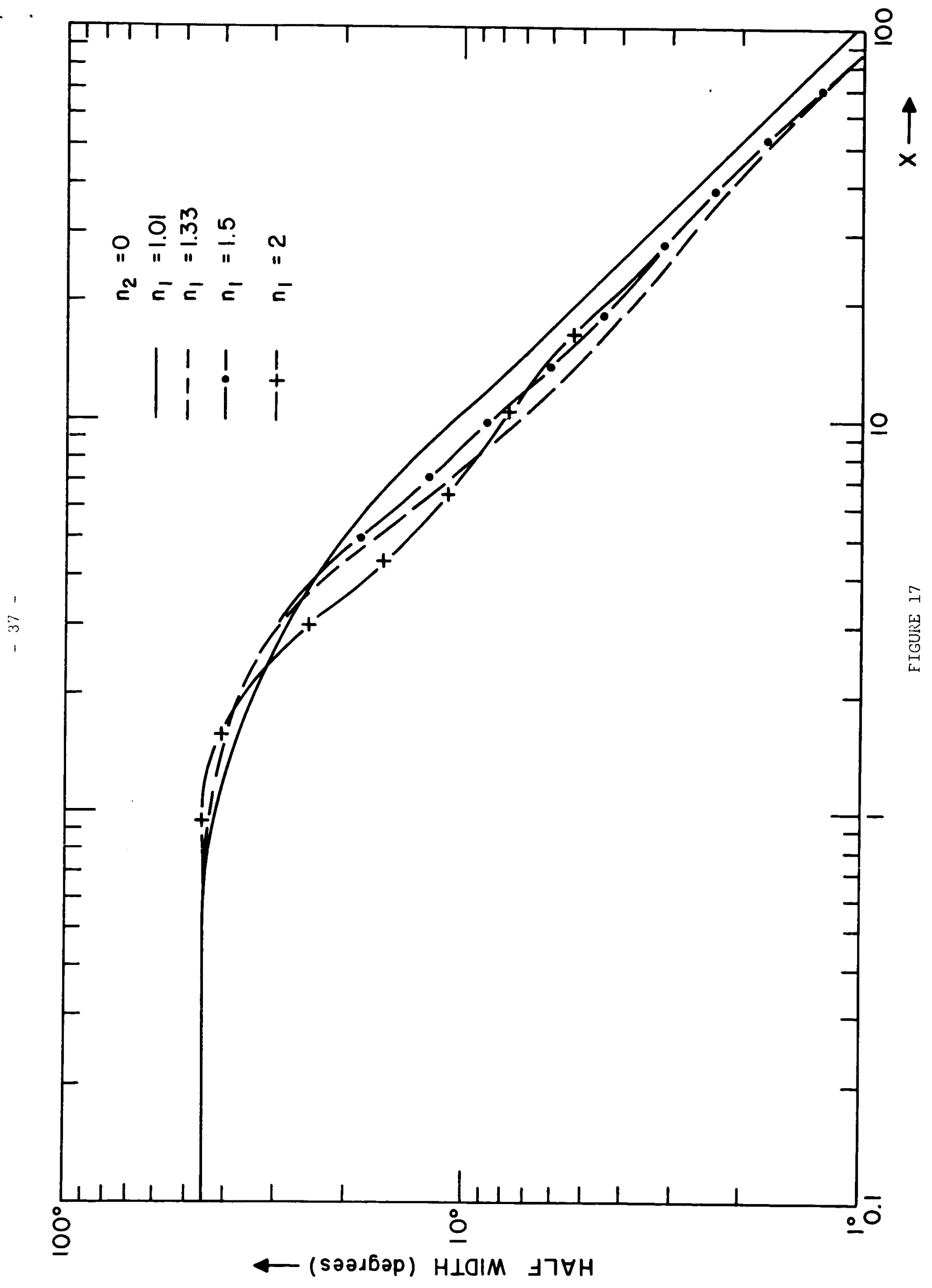




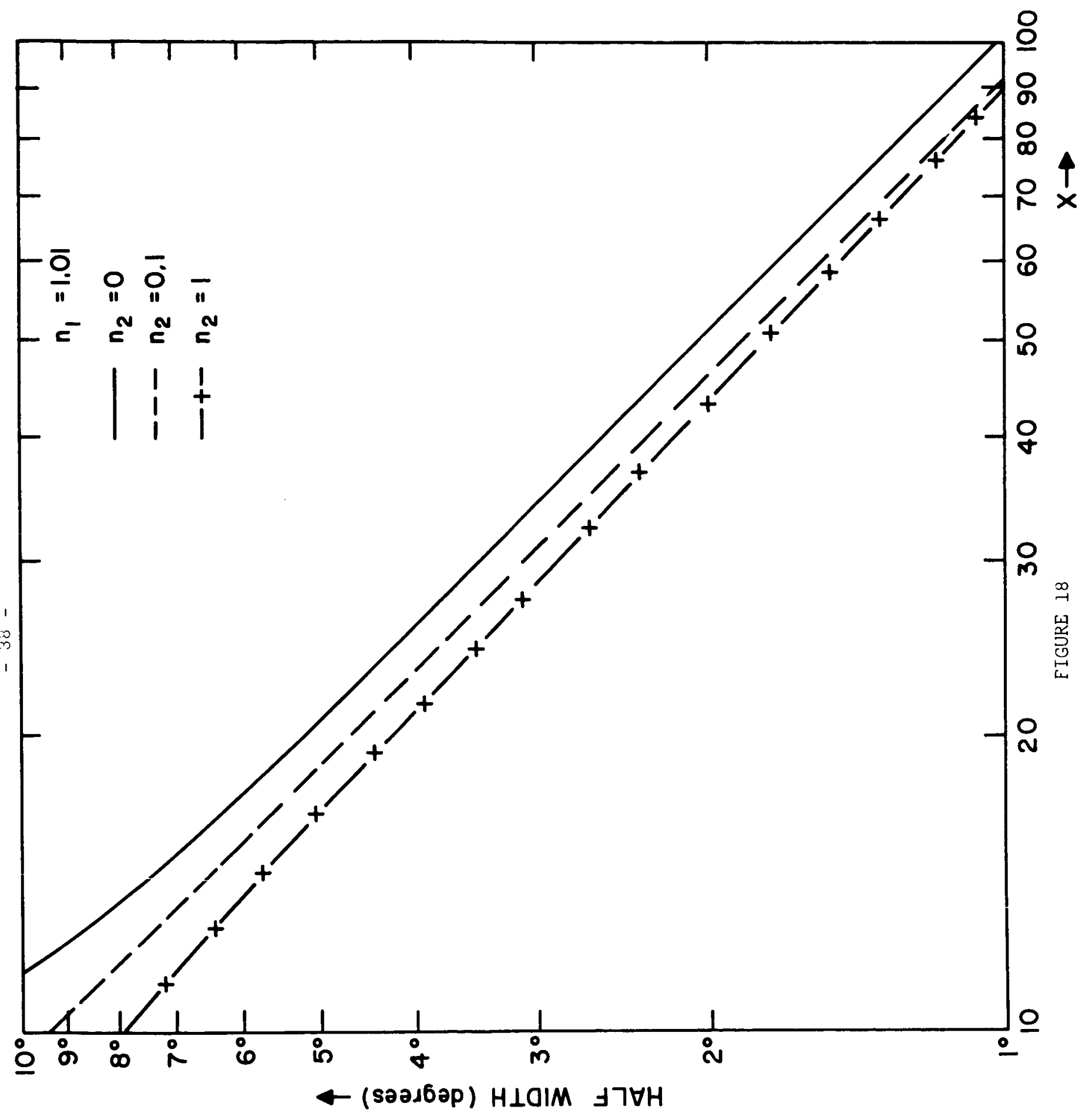




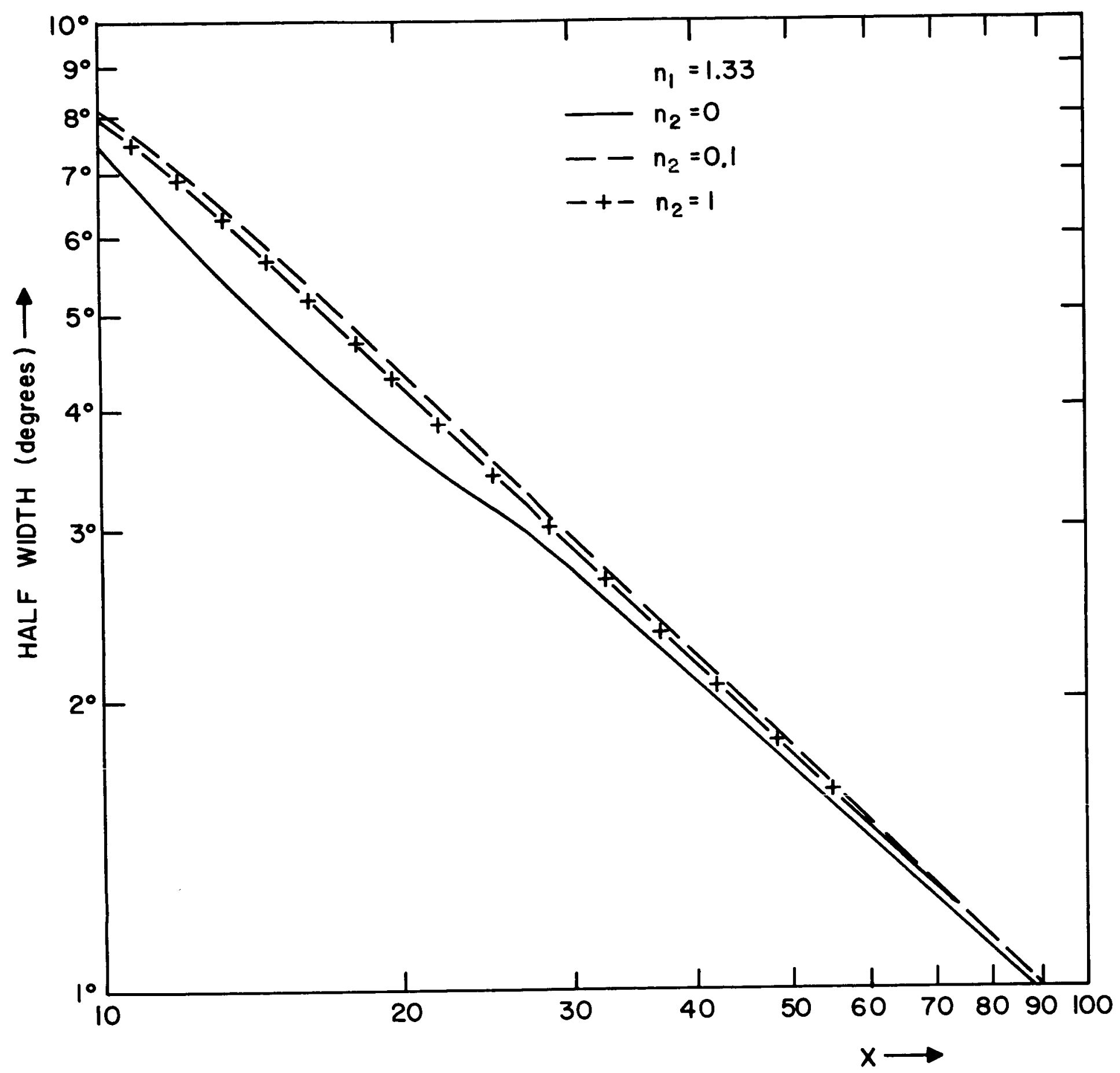


References

1. H. C. Van de Hulst, Light Scattering by Small Particles (Wiley, New York, 1957).

2. D. Deirmendjian, R. Clasen, and W. Viezee, J. Opt. Soc. Am. 51, 620 (1961).

3. A. Brockes, Optik 2I, 550 (1964).

4. G. N. Plass, Appl. Opt. 5 , 279 (1966).

5. A. L. Aden, J. Appl. Phys. 22, 601 (1951).

6. F. T. Corbato and T. L. Uretsky, J. Assoc. for Computing Machinery 6, $366(1959)$.

7. R. H. Giese, Z. Astrophy., 51, 119 (1961).

8. P. J. Wyatt, Phys. Rev. 127, 1837 (1962).

9. L. Infeld, Quart. Appl. Math., 5, 113 (1947).

10. W. M. Irvine, J. Opt. Soc. Amer. 55, 16 (1965). 


\section{Captions for Figures}

Fig. 1. Efficiency factor for absorption as a function of $\mathrm{n}_{2}$ for $x=2 \pi a / \lambda=0.1$ and various values of $n_{1}$.

Fig. 2. Efficiency factor for absorption as a function of $n_{2}$ for $\mathrm{x}=1$ and various values of $\mathrm{n}_{1}$.

Fig. 3. Efficiency factor for absorption as a function of $n_{2}$ for $\mathrm{x}=10$ and various values of $\mathrm{n}_{1}$.

Fig. 4. Efficiency factor for scattering as a function of $n_{2}$ for $\mathrm{x}=0.1$ and various values of $\mathrm{n}_{1}$.

Fig. 5. Efficiency factor for scattering as a function of $n_{2}$ for $\mathrm{x}=1$ and various values of $\mathrm{n}_{1}$.

Fig. 6. Efficiency factor for scattering as a function of $\mathrm{n}_{2}$ for $\mathrm{x}=10$ and various values of $\mathrm{n}_{1}$.

Fig. 7. Maximum value of $\mathrm{Q}_{\text {ext }}$ at the first resonance as a function of $n_{1}$ for various values of $n_{2}$.

Fig. 8. Width at half-maximum (in units of $x$ ) as a function of $n_{1}$ for various values of $n_{2}$.

Fig. 9. Average value of the cosine of the scattering angle as a function of $n_{2}$ for $x=0.1$ and various values of $n_{1}$.

Fig. 10. Average value of the cosine of the scattering angle as a function of $n_{2}$ for $x=l$ and various values of $n_{1}$.

Fig. 11. Average value of the cosine of the scattering angle as a function of $n_{2}$ for $x=10$ and various values of $n_{1}$. 
Fig. 12. Average value of the cosine of the scattering angle and $Q_{\text {ext }}$ as a function of $x$ for $n_{1}=10$ and $n_{2}=0$. The scales for the cosine and for $Q_{\text {ext }}$ are on the left and right respectively.

Fig. 13. Average value of the cosine of the scattering angle and $Q_{\text {ext }}$ as a function of $x$ for $n_{1}=10$ and $n_{2}=0$ in the vicinity of the second and third resonances. The scales for the cosine and for $\mathrm{Q}_{\text {ext }}$ are on the left and right respectively.

Fig. 14. Average value of the cosine of the scattering angle as a function of the size parameter $\mathrm{x}$ for $\mathrm{n}_{1}=10$ and 100. These values of $\mathrm{x}$ are in the immediate neighborhood of the first resonance in $Q_{\text {ext }}$.

Fig. 15. The scattered intensity as a function of the scattering angle for $x=0.275,0.31131$, and 0.360 and $n_{1}=10, n_{2}=0$. The values of the intensity at $0^{\circ}$ and $180^{\circ}$ are indicated above the curves near each margin. The solid curve is the intensity il (as defined by Van de Hulst ${ }^{l}$ ) and the dashed curve is the intensity $i_{2}$. The logarithm of the intensity is plotted; each division indicated on this scale represents a factor of ten.

Fig. 16. The scattered intensity as a function of the scattering angle for $x=0.44404,0.44775,0.57330$, and 0.57516 which corresponds to the electric dipole, magnetic quadrupole, electric quadrupole, and magnetic octopole resonances respectively and for $\mathrm{n}_{1}$ - $10, \mathrm{n}_{2}=0$. See legend for Fig. 15 .

Fig. 17. Half-width of angular intensity function in degrees as a function of size parameter $x$ for $n_{2}=0$. 
Fig. 18. Half-width of angular intensity function in degrees as a function of size parameter $\mathrm{x}$ for $\mathrm{n}_{1}=1.01$.

Fig. 19. Half-width of angular intensity functions in degrees as a function of size parameter $x$ for $n_{1}=1.33$. 
Unclassified

Security Classification

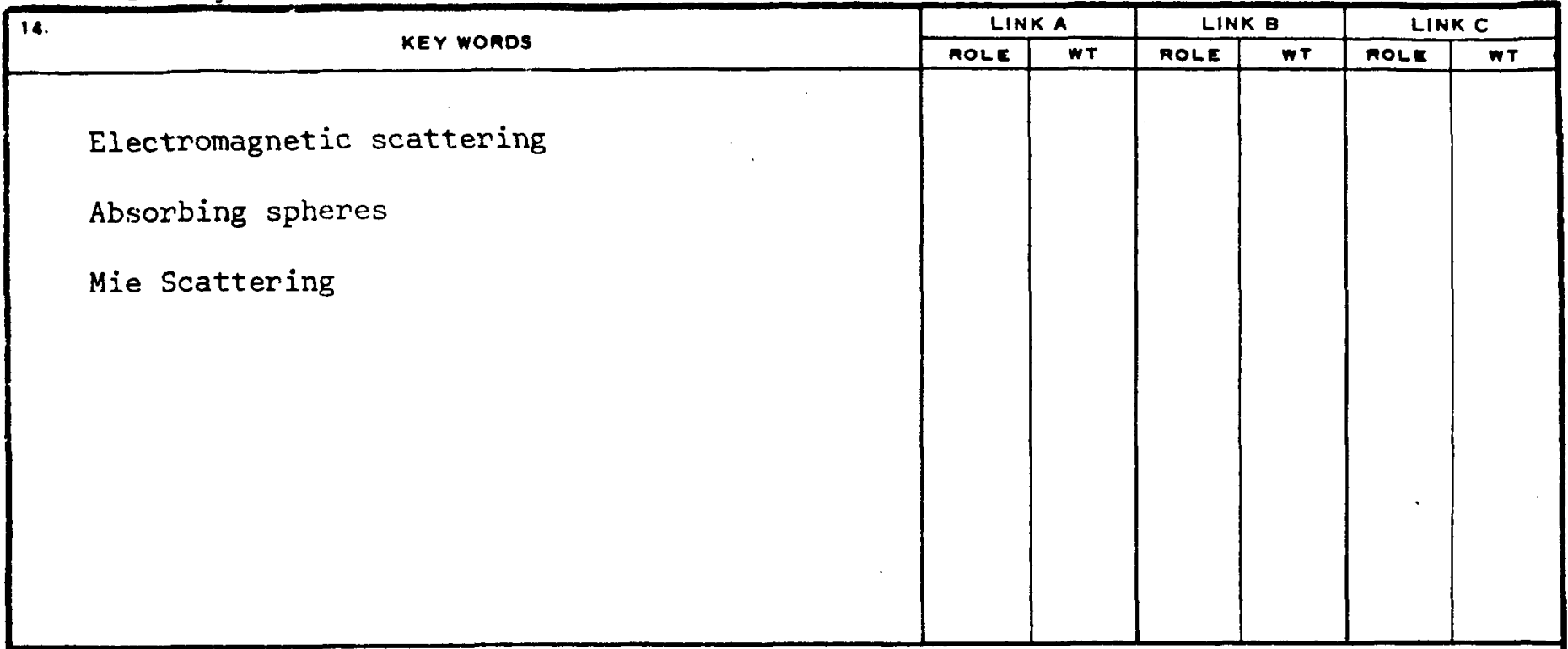

\section{INSTRUCTIONS}

1. ORIGINATING ACTIVITY: Enter the name and address of the contractor, subcontractor, grantee, Department of Defense activity or other organization (corporate author) issuing the report.

2a. REPORT SECURTY Y CLASSIFICATION: Enter the overall security classification of the report. Indic ate whether "Restricted Data" is included. Marking is to be in accordance with appropriate security regulations.

2b. GROUP: Automatic downgrading is specified in DoD DIrective 5200.10 and Armed Forces Industrial Manual. Enter the group number. Also, when applicable, show that optional markings have been used for Group 3 and Group 4 as authorized.

3. REPORT TITLE: Enter the complete report title in all capital letters. Titles in all cases should be unclassified. If a meaningful title cannot be selected without classification, shcw title classification in all capitals in parenthesis immediately following the title.

4. DESCRIPTIVE NOTES: If appropriate, enter the type of report, e.g. int erim, progress, summary, annual, or final.

Give the inclusive dates when a specific reporting period is covered.

5. AUTHOR(S): Enter the name(s) of author(s) as shown on or in the report. Ent et last name, fir st name, middle initial. If rit:iary, show rank and hranch of service. The name of the principal aithor is an abolute minimum requirement.

6. REPORT DAT: Enter the date of the report as day, month, year; or month, year. If more than one date appears or. the report, use date of publication.

7a. TOTAL NUMBER OF PAGES: The total page count shouid follow normal pagination procedures, i.e., enter the number of pages containing information

7b. NUMBER OF REFERENCES. Enter the total number of references cited in the report.

8a. CONTRACT OR GRANT NUMBER: If appropriate, enter the applicable number of the contract or grant under which the report was written

8b, ac, of 8d. PROJECT NUMBER: Enter the appropriate military department identification, such as project number, subproject number, system numbers, task number, etc.

9a. ORIGINATOR'S REPORT NUMBER(S): Enter the official report number by whlch the document will be Identified and controlled by the originating activity. This number must be unique to this report.

9b. OTHER REPORT NUMBER(Ș): If the report has been aseigned any other report numbers (either by the orlginator or by the sponsor), also enter this number(s).

10. AVAIL ABILITY/LIMITATION NOTICES: Enter any limitations on further dissemination of the report, other than those imposed by security classification, using standard statemente auch av:

(1) "Qualified requesters may obtain copies of this report from DDC"

(2) "Foreign announcement and dissemination of this report by DDC is not authorized."

(3) "U. S. Government agencies may obtain copies of this report directly from DDC. Other qualified DDC users shall request through

(4) "U. S. military agencies may obtain copies of this report directly from DDC. Other qualified users shall request through

(5) "All distribution of this report is controlled Qualified DDC users shall request through

.

If the report has been furnished tc the Office of Technical Services, Department of Commerce, for sale to the public, indicate this fact and enter the price, if known

11. SUPPLEMENTARY NOTES: $>$ Use for additional explanatory notes.

12. SPONSORING MILITARY ACTIVITY: Ent er the name of the departmental project office or laboratory sponsoring (paying for) the research and development. Include address.

13. ABSTRACT: Enter an abstract giving a brief and factual summary of the document indicative of the report, even though it may also appear elsewhere in the body of the technical report. If additional space is required, a continuation sheet shall be attached.

It is highly desirable that the abstract of classified reports be unclassified. Each paragraph of the abstract shall end with an indication of the military security classification of the information in the paragraph, represented as (TS), (S), (C), or (U)

There is no limitation en the length of the abstract. How ever, the suggested length is from 150 to 225 words.

14. KEY WORDS: Key words are technically meaningful terms or short phrases that characterize a report and $m$ ay be used as index entries for cataloging the report. Key words must be solected so that no securlty classification in required. Identifiers, such as equipment model designation, trade name, military project code name, geographic location, may be used as key words but will be followed by an indication of technical context. The assimment of links, rules, and weights la optional. 
Unciassified

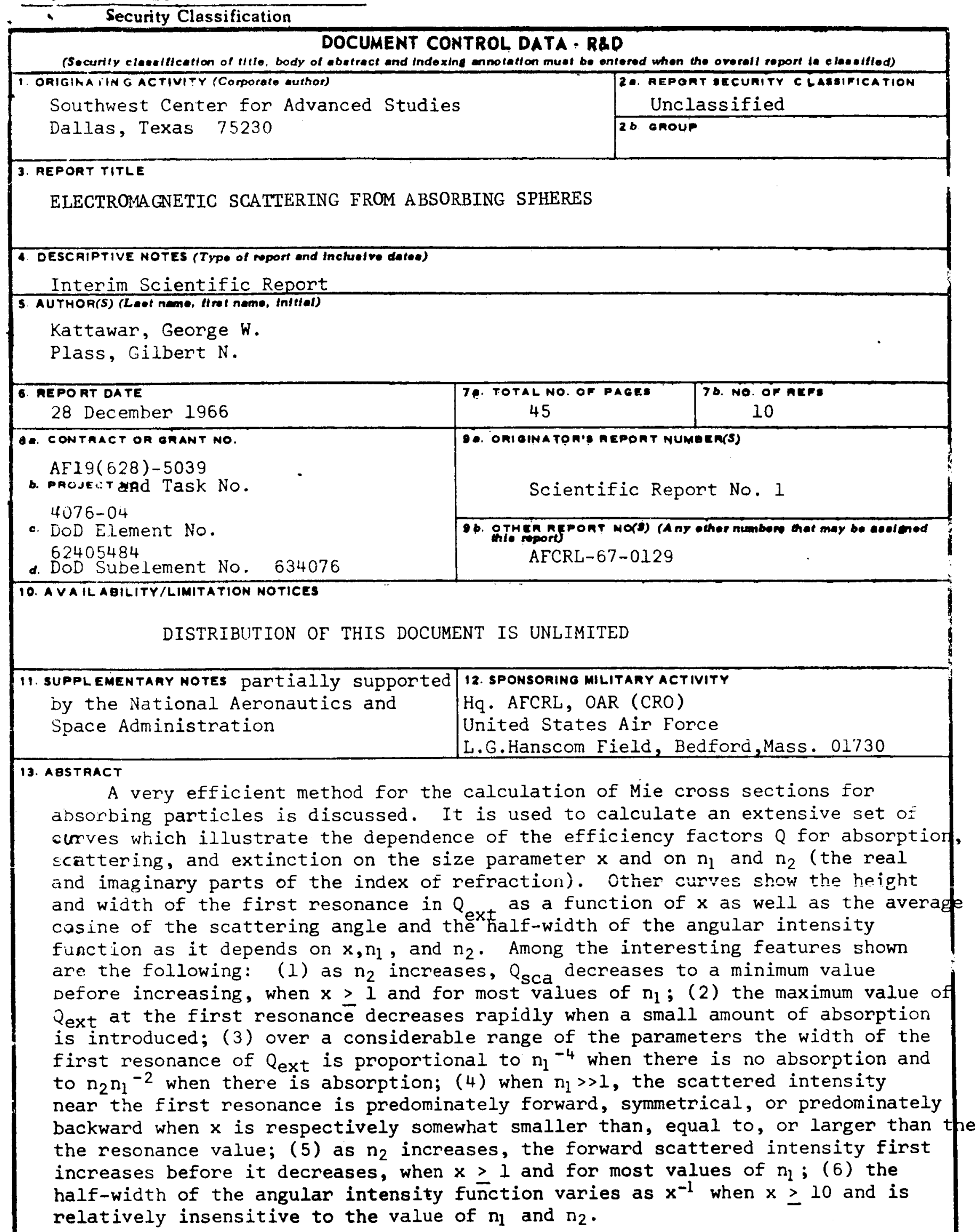

